TRANSACTIONS OF THE

AMERICAN MATHEMATICAL SOCIETY

Volume 351, Number 11, Pages 4381-4409

S 0002-9947(99)02357-0

Article electronically published on April 20, 1999

\title{
ARITHMETICALLY BUCHSBAUM DIVISORS ON VARIETIES OF MINIMAL DEGREE
}

\author{
UWE NAGEL
}

\begin{abstract}
In this paper we consider integral arithmetically Buchsbaum subschemes of projective space. First we show that arithmetical Buchsbaum varieties of sufficiently large degree have maximal Castelnuovo-Mumford regularity if and only if they are divisors on a variety of minimal degree. Second we determine all varieties of minimal degree and their divisor classes which contain an integral arithmetically Buchsbaum subscheme. Third we investigate these varieties. In particular, we compute their Hilbert function, cohomology modules and (often) their graded Betti numbers and obtain an existence result for smooth arithmetically Buchsbaum varieties.
\end{abstract}

\section{INTRODUCTION}

In this paper a variety $X \subset \mathbb{P}^{n}$ will always mean a nondegenerate integral projective subscheme over an algebraically closed field $K$. Let $d$ and $c$ denote its degree and its codimension, respectively. Then $d>c$. If $d=c+1$, then $X$ is said to be a variety of minimal degree.

It is well-known that varieties, which are contained in a variety $V$ of minimal degree as subschemes of codimension one, behave often extremally with respect to various properties. For example, they have maximal geometric genus [11]. We consider these subschemes of codimension one as divisors on $V$.

Our first goal is to characterize the arithmetically Buchsbaum subschemes with maximal Castelnuovo-Mumford regularity as divisors on a variety of minimal degree. Recall that $X$ is $s$-regular if $H^{i}\left(\mathbb{P}^{n}, \mathcal{J}_{X}(s-i)\right)=0$ for all $i \geq 1$. The Castelnuovo-Mumford regularity reg $X$ is the least such integer $s$. The interest in this concept stems partly from the fact that $X$ is $s$-regular if and only if for every $j \geq 0$ the minimal generators of the $j$-th syzygy module of the homogeneous ideal $I(X)$ of $X$ occur in degree $\leq s+j$. In particular, we have $m(X) \leq \operatorname{reg} X$ where $m(X)$ denotes the maximal degree of a minimal generator of $I(X)$. In general, it is rather difficult to prove good upper bounds for $m(X)$ and $\operatorname{reg} X$ in terms of simple invariants of $X$. However, if $X$ is arithmetically Cohen-Macaulay the problem is much better understood thanks to recent investigations (cf., for example, [25], [26], [21], [17]). If $a, b$ are positive integers, let $\left\lceil\frac{a}{b}\right\rceil$ denote the smallest integer $\geq \frac{a}{b}$. A starting point for this paper is the following result.

Received by the editors August 27, 1997.

1991 Mathematics Subject Classification. Primary 14M05; Secondary 13 H10.

Key words and phrases. Minimal generator, local cohomology, Castelnuovo-Mumford regularity, arithmetically Buchsbaum scheme, rational normal scroll.

The material of this paper is part of the author's Habilitationsschrift [18]. 
Theorem 1.1. If $X$ is an arithmetically Cohen-Macaulay variety, then

(a) $\operatorname{reg} X \leq\left\lceil\frac{d-1}{c}\right\rceil+1$.

(b) $m(X) \leq\left\lceil\frac{d}{c}\right\rceil$.

(c) Suppose char $(K)=0$ or $c \geq 5$. If $d>(c+1)^{2}$, then the following conditions are equivalent:

(i) $\operatorname{reg} X=\left\lceil\frac{d-1}{c}\right\rceil+1$

(ii) $m(X)=\left\lceil\frac{d}{c}\right\rceil$.

(iii) $X$ is a divisor on a variety of minimal degree.

In case $\operatorname{char}(K)=0$ the result has been proved in [26]. The general case is due to [17].

Since smooth rational curves with a $(d-c+1)$-secant have Castelnuovo-Mumford regularity $d-c+1$ (cf. [9]) one cannot hope for a generalization of the theorem to arbitrary varieties. One has to restrict himself to arithmetically Buchsbaum varieties as the following result of Stückrad and Vogel [23] indicates.

Theorem 1.2. If $X$ is an arithmetically Buchsbaum variety, then

$$
\operatorname{reg} X \leq\left\lceil\frac{d-1}{c}\right\rceil+1
$$

This is the same bound as in Theorem 1.1. Strictly speaking it is proved in [23] with the additional assumption that $\operatorname{char} K=0$. We show that this assumption can be removed. Furthermore, we prove that equality in the estimate of Theorem 1.2 holds if $X$ is a divisor on a variety of minimal degree and that the estimate can be improved otherwise (cf. Proposition 4.3). As a consequence we obtain.

Theorem 1.3. Let $X$ be an arithmetically Buchsbaum variety of degree $d>$ $\max \{2 c(c+1), 14\}$. Suppose either $\operatorname{char}(K)=0$ or $c \geq 5$. Then the following conditions are equivalent:

(a) $\operatorname{reg} X=\left\lceil\frac{d-1}{c}\right\rceil+1$.

(b) $m(X) \geq\left\lceil\frac{d}{c}\right\rceil$.

(c) $X$ is a divisor on a variety of minimal degree.

Theorem 1.2 provides $m(X) \leq \operatorname{reg} X \leq\left\lceil\frac{d-1}{c}\right\rceil+1$. If we compare this estimate with Theorem 1.1, one might wonder if it can be improved to $m(X) \leq\left\lceil\frac{d}{c}\right\rceil$. However, simple examples show that the latter estimate is not true for every arithmetically Buchsbaum variety $X$. Nevertheless it is very natural to ask if it were true provided the variety has sufficiently large degree. In order to answer this question much more detailed information on the corresponding varieties is necessary. According to Theorem 1.3 we have to study arithmetically Buchsbaum divisors on a variety $V$ of minimal degree. Recall that a variety of minimal degree is either a rational normal scroll, a cone over a quadric hypersurface of rank $\geq 5$ or a cone over the Veronese surface in $\mathbb{P}^{5}$. A modern exposition of this famous classification result can be found in [6]. Using this classification it turns out that on a variety $V$ of minimal degree an integral arithmetically Buchsbaum divisor, which is not 
arithmetically Cohen-Macaulay, does exist only in the case that $V$ is a rational normal scroll.

A rational normal scroll $S \subset \mathbb{P}^{n}$ of dimension $k+1$ and degree $c=n-k$ is up to projective equivalence uniquely determined by a sequence of integers $\left(a_{0}, \ldots, a_{k}\right)$ where $0 \leq a_{0} \leq \ldots \leq a_{k}$ and $a_{0}+\ldots+a_{k}=c$ (cf. Section 5). We write $S=$ $S\left(a_{0}, \ldots, a_{k}\right)$. The divisor class group of $S$ is freely generated by the hyperplane section $H$ and a linear subspace $F \subset S$ of dimension $k$.

A starting point of our investigations of divisors on scrolls is the following wellknown result: An integral space curve $C$, which is a divisor of type $(a, b)$ on a smooth quadric $S \subset \mathbb{P}^{3}$, is arithmetically Buchsbaum but not arithmetically CohenMacaulay if and only if $|a-b|=2$. Here $S$ is just the scroll $S(1,1)$. Thus this statement is a very special case of our main result. It describes the scrolls and divisor classes which contain arithmetically Buchsbaum varieties.

Theorem 1.4. Let $X \subset \mathbb{P}^{n}$ be a Cohen-Macaulay variety being a divisor on a rational surface scroll $S\left(a_{0}, \ldots, a_{k}\right)$. Write $d=t c+1-p$ where $1 \leq p \leq c=n-k$. Then $X$ is arithmetically Buchsbaum but not arithmetically Cohen-Macaulay if and only if either

$X \sim(t-1) H+(1+c-p) F$ and $a_{i} \geq c-p$ for all $i=0, \ldots, k$ or

$X \sim(t+1) H+(1-c-p) F$ and $a_{i} \geq p$ for all $i=0, \ldots, k$.

Moreover, there is a smooth arithmetically Buchsbaum variety in any of these divisor classes.

In particular, it turns out that a singular rational normal scroll does not contain arithmetically Buchsbaum divisors which are not arithmetically Cohen-Macaulay. We also compute the Hilbert function and the cohomology of the divisors mentioned in the theorem above and obtain information on the minimal free graded resolution of their defining ideal.

As a consequence of our considerations we conclude that there are triples $(d, c, k)$ for which there is no corresponding arithmetically Buchsbaum variety, which is not arithmetically Cohen-Macaulay but a divisor on a scroll. This is in contrast to the case of arithmetically Cohen-Macaulay varieties. The precise existence result is as follows.

Theorem 1.5. Suppose we are given integers $d, c, k$ where $d>c, c \geq 2, k \geq 1$. Write $d=t c+1-p$ where $1 \leq p \leq c=n-k$. Then the following conditions are equivalent:

(a) There is an arithmetically Buchsbaum variety $X \subset \mathbb{P}^{c+k}$ of degree $d$, codimension $c$ and dimension $k$ being a divisor on a rational normal scroll but not arithmetically Cohen-Macaulay.

(b) There is a smooth variety having the properties described in (a).

(c) It holds that $1 \leq p \leq \frac{c}{k+1}$ or $\frac{k}{k+1} c \leq p<c$.

Moreover, if $1 \leq p \leq \frac{c}{k+1}$, then we are able to determine all graded Betti numbers of such an arithmetically Buchsbaum variety. In particular, it turns out that in this case we have $m(X)=\left\lceil\frac{d}{c}\right\rceil+1$. That is, we cannot hope for an analogue of Theorem 1.1,(b) for arithmetically Buchsbaum varieties just by requiring that the degree is large enough. However, there is a good deal of information on the varieties for which the estimate fails. 
The paper is organized as follows. In Section 2 we compute the graded Betti numbers of reduced arithmetically Cohen-Macaulay divisors on a variety of minimal degree (cf. Theorem 2.4). Moreover, we show that integral (locally) CohenMacaulay divisors on a quadric of rank $\geq 5$ and on a cone over the Veronese surface are arithmetically Cohen-Macaulay (cf. Proposition 2.9); thus we know their graded Betti numbers. In Section 3 we show a lifting result which yields that an integral variety must be a divisor on a variety of minimal degree if its general hyperplane section has this property (cf. Proposition 3.3). Combined with Theorem 2.4 it implies preliminary information on the cohomology modules of reduced arithmetically Buchsbaum divisors on a variety of minimal degree (cf. Proposition 3.6). Section 4 is devoted to Castelnuovo bounds. It contains the proof of Theorem 1.3. In the final section we put together techniques of [11] and our previous results in order to study divisors on rational normal scrolls. First we derive necessary conditions for the possible divisor classes which contain arithmetically Buchsbaum varieties (cf. Lemma 5.6) and then also for the scrolls. Here, in particular the results on the cohomology of Section 3 play a crucial role. Second we show that the necessary conditions are also sufficient. The main result is Theorem 5.10. It covers Theorem 1.4 and contains in addition results on the cohomology and Betti numbers of the considered varieties. Moreover, it implies Theorem 1.5 rather quickly.

For background information concerning arithmetically Buchsbaum schemes we refer to the monograph [24]. Throughout this paper $R$ will denote the polynomial ring $K\left[x_{0}, \ldots, x_{n}\right]$ and $\mathfrak{m}=\left(x_{0}, \ldots, x_{n}\right)$ its maximal homogeneous ideal. Moreover we always assume that the subscheme $X \subset \mathbb{P}^{n}$ has codimension $c \geq 2$. This is not a restriction of generality because the results are trivial if $X$ has codimension 1 .

\section{Arithmetically Cohen-Macaulay divisors ON A VARIETY OF MINIMAL DEGREE}

A starting point for the investigations of arithmetically Buchsbaum varieties in this paper are the corresponding results for arithmetically Cohen-Macaulay varieties which have been obtained recently. A sample result is the following (cf. [25], [21]).

Theorem 2.1. Let $X$ be an arithmetically Cohen-Macaulay variety. Suppose $\operatorname{char}(K)=0$ or $c \geq 5$. If $d>(c+1)^{2}$, then the following conditions are equivalent:

(a) $\operatorname{reg} X=\left\lceil\frac{d-1}{c}\right\rceil+1$.

(b) $m(X)=\left\lceil\frac{d}{c}\right\rceil$.

(c) $X$ is a divisor on a variety of minimal degree.

Varieties satisfying the conditions of the theorem exist in abundance. For example, any smooth Castelnuovo variety will do. In this section we compute the graded Betti numbers of these varieties. Then we discuss the range of applications of this result.

We begin by studying the resolution of a finite subscheme $X \subset \mathbb{P}^{n}$ in linearly general position lying on a rational normal curve where $\operatorname{deg} X>2 n$. In case $\operatorname{deg} X \leq 2 n+1$ this has already been done in [19], Proposition 2.3.

Recall that a finite subscheme $Z \subset \mathbb{P}^{n}$ is said to be in linearly general position if for any proper linear subspace $N$ of $\mathbb{P}^{n}$ we have

$$
\operatorname{deg} X \cap N \leq \operatorname{dim} N+1 \text {. }
$$


Proposition 2.2. Let $X$ be a finite subscheme in linearly general position lying on a rational normal curve of $\mathbb{P}^{n}$. Suppose $\operatorname{deg} X>2 n$. Then the homogeneous ideal of $X$ has a minimal free graded resolution of the following form:

$$
0 \rightarrow F_{n} \rightarrow \ldots \rightarrow F_{1} \rightarrow I(X) \rightarrow 0
$$

where

$$
\begin{gathered}
F_{i}=R(-1-i)^{\alpha_{i}} \oplus R(-t+1-i)^{\beta_{i}} \oplus R(-t-i)^{\gamma_{i}} \quad(i=1, \ldots, n), \\
d=\operatorname{deg} X=t n+1-p \quad \text { with } 1 \leq p \leq n
\end{gathered}
$$

and

$$
\begin{gathered}
\alpha_{i}=i\left(\begin{array}{c}
n \\
i+1
\end{array}\right), \\
\beta_{i}=\left\{\begin{array}{lll}
p\left(\begin{array}{c}
n \\
i-1
\end{array}\right)-n\left(\begin{array}{c}
n-1 \\
i-2
\end{array}\right) & \text { if } 1 \leq i \leq p, \\
0 & \text { if } \quad p<i \leq n,
\end{array}\right. \\
\gamma_{i}=\left\{\begin{array}{lll}
0 & \text { if } 1 \leq i \leq p, \\
n\left(\begin{array}{c}
n-1 \\
i-1
\end{array}\right)-p\left(\begin{array}{c}
n \\
i
\end{array}\right) & \text { if } & p<i \leq n .
\end{array}\right.
\end{gathered}
$$

Proof. Since $X$ is in linearly general position, we get by [5], Theorem 3.2 for the Hilbert function of $X$ that $h_{X}(j) \geq \min \{d, j n+1\}$ if $j \geq 0$. Let $V$ be the rational normal curve containing $X$. Then $h_{X}(j) \leq h_{V}(j)=j n+1$ if $j \geq 0$. It follows $h_{X}(j)=\min \{d, j n+1\}$. Moreover, we get for the Hilbert function of $M=I(X) / I(V)$

$$
h_{M}(j)= \begin{cases}0 & \text { if } \quad i<t \\ (j-t) n+p & \text { if } i \geq t .\end{cases}
$$

In particular, we have $h_{M}(t)=p$. Since $M$ is torsion-free as $R / I(V)$-module [7], Theorem 1.1 gives

$$
\left[\operatorname{Tor}_{i}^{R}(K, M)\right]_{j}=0 \quad \text { if } j<i+t
$$

and

$$
\left[\operatorname{Tor}_{i}^{R}(K, M)\right]_{i+t}=0 \quad \text { if } i \geq p .
$$

Let us consider the exact sequence

$$
\begin{aligned}
(*) \operatorname{Tor}_{i+1}^{R}(K, M) \rightarrow \operatorname{Tor}_{i}^{R}(K, I(V)) \rightarrow \operatorname{Tor}_{i}^{R}(K, I(X)) & \rightarrow \operatorname{Tor}_{i}^{R}(K, M) \\
& \rightarrow \operatorname{Tor}_{i-1}^{R}(K, I(V)) .
\end{aligned}
$$

The resolution of a rational normal curve is well-known. In particular, we have (cf., for example, [19], Lemma 2.2)

$$
\operatorname{rank}\left[\operatorname{Tor}_{i}^{R}(K, I(V))\right]_{j}= \begin{cases}(i+1)\left(\begin{array}{c}
n \\
i+2
\end{array}\right) & \text { if } j=i+2 \text { and } 0 \leq i \leq n-2, \\
0 & \text { otherwise. }\end{cases}
$$

By assumption we have $t \geq 3$. Therefore, the exact sequence $(*)$ implies for $i \geq 0$

$$
\alpha_{i+1}=\operatorname{rank}\left[\operatorname{Tor}_{i}^{R}(K, I(X))\right]_{i+2}=\operatorname{rank}\left[\operatorname{Tor}_{i}^{R}(K, M)\right]_{i+2}=(i+1)\left(\begin{array}{c}
n \\
i+2
\end{array}\right)
$$

and

$$
\left[\operatorname{Tor}_{i}^{R}(K, I(X))\right]_{j} \cong\left[\operatorname{Tor}_{i}^{R}(K, M)\right]_{j} \quad \text { if } j \neq i+1, i+2 .
$$


From the Hilbert function of $X$ we see that $\operatorname{reg} X \leq t+1$ and therefore

$$
\left[\operatorname{Tor}_{i}^{R}(K, I(X))\right]_{j}=0 \text { if } j \geq i+t+2
$$

(cf., for example, [19], Corollary 1.4). Hence, we obtain

$$
\left[\operatorname{Tor}_{i}^{R}(K, M)\right]_{j}=0 \quad \text { if } j \neq i+t, i+t+1 .
$$

Thus, the claim is shown if we have computed

$$
\begin{aligned}
& \beta_{i+1}=\operatorname{rank}\left[\operatorname{Tor}_{i}^{R}(K, I(X))\right]_{i+t}=\operatorname{rank}\left[\operatorname{Tor}_{i}^{R}(K, M)\right]_{i+t} \text { and } \\
& \gamma_{i+1}=\operatorname{rank}\left[\operatorname{Tor}_{i}^{R}(K, I(X))\right]_{i+t+1}=\operatorname{rank}\left[\operatorname{Tor}_{i}^{R}(K, M)\right]_{i+t+1} .
\end{aligned}
$$

To this end we want to use Hilbert series. Let $Q$ be a finitely generated graded $R$-module and let $Z$ be an indeterminate. Let us denote the Hilbert function of $Q$ by $h_{Q}$. Then the Hilbert series of $Q$ is defined as $H(Q, Z)=\sum_{j \geq 0} h_{Q}(j) Z^{j}$.

Let $h: \mathbb{Z} \rightarrow \mathbb{Z}$ be a numerical function. The first difference function $\Delta h$ is defined by $\Delta h(j)=h(j)-h(j-1)$. For a non-negative integer $s$ we define $\Delta^{s} h$ by $\Delta^{0} h=h$ and $\Delta^{s} h=\Delta\left(\Delta^{s-1} h\right)$ if $s>0$.

If $Q$ is a module of dimension $r$, then it is well-known and easy to see that

$$
H(Q, Z)=\frac{\sum_{j \geq 0} \Delta^{r} h_{Q}(j) Z^{j}}{(1-Z)^{r}}
$$

where the sum in the numerator is in fact finite. Hence, the Hilbert series of $R(-e)^{a}$ is $\frac{a Z^{e}}{(1-Z)^{n+1}}$.

Let us come back to the computation of the Betti numbers of $M$. We already know that $M$ has a minimal free graded resolution of the form

$$
0 \rightarrow R(-t-n)^{\gamma_{n}} \oplus R(-t-n+1)^{\beta_{n}} \rightarrow \ldots \rightarrow R(-t-1)^{\gamma_{1}} \oplus R(-t)^{\beta_{1}} \rightarrow M \rightarrow 0 .
$$

Thus, using the additivity of $\operatorname{rank}_{K}$ on exact sequences we get

$$
H(M, Z)=\frac{\sum_{j=1}^{n+1}(-1)^{j+1}\left(\beta_{j}-\gamma_{j-1}\right) Z^{t-1+j}}{(1-Z)^{n+1}}
$$

where we define $\beta_{n+1}=\gamma_{0}=0$. Due to the result above on the Hilbert function of $M$ we obtain

$$
H(M, Z)=\frac{\sum_{j \geq 0} \Delta^{2} h_{M}(j)}{(1-Z)^{2}}=\frac{p Z^{t}+(n-p)^{t+1}}{(1-Z)^{2}} .
$$

It follows

$$
\sum_{j=1}^{n+1}(-1)^{j+1}\left(\beta_{j}-\gamma_{j-1}\right) Z^{t-1+j}=\left(p Z^{t}+(n-p) Z^{t+1}\right)(1-Z)^{n-1} .
$$

Comparing coefficients gives

$$
(-1)^{j+1)}\left(\beta_{j}-\gamma_{j-1}\right)=(-1)^{j-1} p\left(\begin{array}{c}
n-1 \\
j-1
\end{array}\right)+(-1)^{j}(n-p)\left(\begin{array}{c}
n-1 \\
j-2
\end{array}\right)
$$

and thus

$$
\beta_{j}-\gamma_{j-1}=p\left(\begin{array}{c}
n \\
j-1
\end{array}\right)-n\left(\begin{array}{c}
n-1 \\
j-2
\end{array}\right) \text { for } j=1, \ldots, n+1 .
$$

By $(+)$ we know that $\beta_{j}=0$ if $j \geq p+1$. If follows

$$
\gamma_{j}=n\left(\begin{array}{c}
n-1 \\
j-1
\end{array}\right)-p\left(\begin{array}{c}
n \\
j
\end{array}\right) \quad \text { if } p \leq j \leq n
$$


In particular, we get $\gamma_{p}=0$. Since $M$ is Cohen-Macaulay this implies $\gamma_{1}=\ldots=$ $\gamma_{p-1}=0$. Hence, we obtain $\beta_{j}=p\left(\begin{array}{c}n \\ j-1\end{array}\right)-n\left(\begin{array}{c}n-1 \\ j-2\end{array}\right)$ if $1 \leq j \leq p$ and the proof is completed.

Remark 2.3. (i) An alternative way to obtain the graded Betti numbers of divisors on a rational curve is indicated in the exercises A2.21 and A3.30 of [3]. It applies to arithmetically Cohen-Macaulay divisors on a rational normal scroll. We have chosen the approach described in the proof above in order to introduce the method which also applies to arithmetically Buchsbaum divisors (cf. Theorem 5.10).

(ii) Still another approach to Proposition 2.2 is due to Lvovski. But his method gives the result only if $1 \leq p \leq 3$ (cf. [15], Propositions 6.1, 6.2 and 6.4).

Using hyperplane sections we can extend our last result in order to treat higherdimensional subschemes.

Theorem 2.4. Let $X \subset \mathbb{P}^{n}$ be a reduced arithmetically Cohen-Macaulay subscheme of degree $d>c=\operatorname{codim} X$. Suppose $X$ is a divisor on a variety of minimal degree. Then the minimal free resolution of the homogeneous coordinate ring $A$ of $X$ has the following shape:

$$
0 \rightarrow F_{c} \rightarrow \ldots \rightarrow F_{1} \rightarrow R \rightarrow A \rightarrow 0
$$

where

$$
\begin{gathered}
F_{i}=R(-1-i)^{\alpha_{i}} \oplus R(-t+1-i)^{\beta_{i}} \oplus R(-t-i)^{\gamma_{i}} \quad(i=1, \ldots, c), \\
d=t c+1-p \quad \text { with } 1 \leq p \leq c
\end{gathered}
$$

and

$$
\begin{gathered}
\alpha_{i}=i\left(\begin{array}{c}
c \\
i+1
\end{array}\right), \\
\beta_{i}= \begin{cases}p\left(\begin{array}{c}
c \\
i-1
\end{array}\right)-c\left(\begin{array}{c}
c-1 \\
i-2
\end{array}\right) & \text { if } 1 \leq i \leq p, \\
0 & \text { if } p<i \leq c,\end{cases} \\
\gamma_{i}=\left\{\begin{array}{lll}
0 & \text { if } 1 \leq i \leq p, \\
c\left(\begin{array}{c}
c-1 \\
i-1
\end{array}\right)-p\left(\begin{array}{c}
c \\
i
\end{array}\right) & \text { if } & p<i \leq c .
\end{array}\right.
\end{gathered}
$$

Proof. Let $L_{c} \subset \mathbb{P}^{n}$ be a general linear subspace of dimension $c$ and let $Y=X \cap L_{c}$. Since $X$ is arithmetically Cohen-Macaulay, the graded Betti numbers of $X \subset \mathbb{P}^{n}$ are the same as those of $Y \subset L_{c} \cong \mathbb{P}^{c}$. Moreover, $Y$ is a reduced zero-dimensional subscheme lying on a rational normal curve. Hence, $Y$ is in linearly general position due to Bezout's theorem and Proposition 2.2 gives the claim if $d>2 c$. If $c+1 \leq$ $d \leq 2 c$, the claim says $\operatorname{rank}\left[\operatorname{Tor}_{i}^{R}(K, A)\right]_{i+1}=\alpha_{i}+\beta_{i}$. Hence the assertion follows by [19], Proposition 2.3 and some manipulation of the formulas given there.

We want to point out two applications of the theorem. Recall that the geometric genus $p_{g}(X)$ of a variety $X \subset \mathbb{P}^{n}$ satisfies

$$
p_{g}(X) \leq\left(\begin{array}{c}
N \\
n-c+1
\end{array}\right) c+\left(\begin{array}{c}
N \\
n-c
\end{array}\right) \varepsilon
$$

where $N$ and $\varepsilon$ are defined by $d-1=N c+\varepsilon$ whereby $0 \leq \varepsilon<c$. This estimation has been shown by Harris [11] in characteristic zero and by Ballico [1] in positive characteristic. If $d=\operatorname{deg} X \geq(n-c) c+2$ and the geometric genus of $X$ achieves 
the bound, then $X$ is called a Castelnuovo variety. We have just computed the graded Betti numbers of smooth Castelnuovo varieties.

Corollary 2.5. Let $X \subset \mathbb{P}^{n}$ be a smooth Castelnuovo variety. Then the resolution of $X$ if of the form as described in Theorem 2.4.

Proof. Theorem 2.4 applies to $X$ by [17], Theorem 4.5 .

Another consequence of our theorem is that in case $X$ is a reduced subscheme being a divisor on a variety of minimal degree we can decide whether $X$ is arithmetically Cohen-Macaulay if we just know the degrees of the defining equations of $X$.

Corollary 2.6. Let $X \subset \mathbb{P}^{n}$ be a reduced subscheme of positive dimension and of degree $d>c$. Write $d=t c+1-p$ where $1 \leq p \leq c$. Suppose $X$ is a divisor on a variety of minimal degree. Then $X$ is arithmetically Cohen-Macaulay if and only if its homogeneous ideal is generated by $\frac{c(c-1)}{2}$ quadrics and $p$ forms of degree $t$ and is generated by $\frac{c(c-1)}{2}+p$ quadrics if $t=2$, respectively.

Proof. If $X$ is arithmetically Cohen-Macaulay, the claim is shown in Theorem 2.4. In order to prove the converse let us assume that $f=\operatorname{depth} A \leq k=\operatorname{dim} X$ where $A$ is the homogeneous coordinate ring of $X$. We have to show that $I(X)$ has a different set of minimal generators as the one described in the statement.

Let $L_{k} \subset \ldots \subset L_{1} \subset L_{0}=\mathbb{P}^{n}$ be a flag of general linear subspaces where $L_{j}$ has dimension $n-j$ and is defined by linear forms $l_{1}, \ldots, l_{j}$. Put $R^{(j)}=R /\left(l_{1}, \ldots, l_{j}\right) R$ and $X_{j}=X \cap L_{j}$ for $j=0, \ldots, k$. Let $A^{(j)}=R^{(j)} / I\left(X_{j}\right)$ be the homogeneous coordinate ring of $X_{j} \subset L_{j}$. Since $A$ has depth $f$, the Betti numbers of $A$ and $A^{(f-1)}$ coincide. In particular, we have

$$
\operatorname{Tor}_{0}^{R}(K, I(X)) \cong \operatorname{Tor}_{0}^{R^{(f-1)}}\left(K, I\left(X_{f-1}\right)\right) .
$$

Moreover, $A^{(f-1)}$ must have depth 1, i.e. $H_{\mathfrak{m}}^{1}\left(A^{(f-1)}\right) \neq 0$. Consider the exact sequence

$$
0 \rightarrow H_{\mathfrak{m}}^{0}\left(A^{(f-1)} / l_{f} A^{(f-1)}\right) \rightarrow H_{\mathfrak{m}}^{1}\left(A^{(f-1)}\right) \stackrel{l_{f}}{\longrightarrow} H_{\mathfrak{m}}^{1}\left(A^{(f-1)}\right) \rightarrow \ldots
$$

Since $H_{\mathfrak{m}}^{1}\left(A^{(f-1)}\right)$ is finitely generated, Nakayama's lemma provides

$$
H_{\mathfrak{m}}^{0}\left(A^{(f-1)} / l_{f} A^{(f-1)}\right) \neq 0 .
$$

Let $a=a\left(H_{\mathfrak{m}}^{0}\left(A^{(f-1)} / l_{f} A^{(f-1)}\right)\right)$. Using

$$
H_{\mathfrak{m}}^{0}\left(A^{(f-1)} / l_{f} A^{(f-1)}\right) \cong I\left(X_{f}\right) /\left(I\left(X_{f-1}\right)+l_{f} R^{(f-1)}\right)
$$

we obtain

$$
\begin{aligned}
{\left[\operatorname{Tor}_{0}^{R^{(f-1)}}\left(K, I\left(X_{f-1}\right)\right)\right]_{j} } & \cong\left[\operatorname{Tor}_{0}^{R^{(f-1)}}\left(K, I\left(X_{f-1}\right)+l_{f} R^{(f-1)}\right)\right]_{j} \\
& \cong\left[\operatorname{Tor}_{0}^{R^{(f)}}\left(K, I\left(X_{f}\right)\right)\right]_{j} \quad \text { if } j<a
\end{aligned}
$$

and

$$
\operatorname{rank}\left[\operatorname{Tor}_{0}^{R^{(f-1)}}\left(K, I\left(X_{f-1}\right)\right)\right]_{a}<\operatorname{rank}\left[\operatorname{Tor}_{0}^{R^{(f)}}\left(K, I\left(X_{f}\right)\right)\right]_{a} .
$$

Applying the same reasoning to $X_{f}, \ldots, X_{k-1}$ we see that there is an integer $b$ such that

$$
\operatorname{rank}\left[\operatorname{Tor}_{0}^{R}(K, I(X))\right]_{b}<\operatorname{rank}\left[\operatorname{Tor}_{0}^{R^{(k)}}\left(K, I\left(X_{k}\right)\right)\right]_{b}
$$


But $X_{k}$ is a set of $d$ points on a rational normal curve. Therefore, we know the minimal free resolution of $I\left(X_{k}\right)$ by Proposition 2.2. It follows that the inequality above contradicts the assumption on the minimal generators of $I(X)$ and we are done.

Remark 2.7. Let us consider the statement above in case $\operatorname{codim} X=2$. It provides: If $X$ has even degree, then $X$ is arithmetically Cohen-Macaulay if and only if $X$ is a complete intersection. If $X$ has odd degree, then $X$ is not a complete intersection but a quasi-complete intersection, i.e., cut out by three hypersurfaces.

Note that as a consequence of the syzygy theorem of Evans and Griffith a smooth variety $X \subset \mathbb{P}^{n}$ of codimension 2 where $n \geq 6$ is projectively normal if and only if it is a complete intersection [8], Theorem 2.3.

According to [6] a variety $V \subset \mathbb{P}^{n}$ of minimal degree is either a rational normal scroll, a quadric of rank $\geq 5$ or a cone over the Veronese surface $W \subset \mathbb{P}^{5}$. Hereby the Veronese surface is considered as a cone over itself. We want to show that smooth integral non-arithmetically divisors on $V$ exist only if $V$ is a rational normal scroll. To this end we recall the following result of Huneke and Ulrich [14].

Lemma 2.8. Let $X \subset \mathbb{P}^{n}$ be a Cohen-Macaulay subscheme of pure dimension $\geq 2$. Then the general hyperplane section of $X$ is arithmetically Cohen-Macaulay if and only if $X$ is arithmetically Cohen-Macaulay.

This result is used to show

Proposition 2.9. Let $X \subset \mathbb{P}^{n}$ be subscheme that is either

(i) an integral divisor on a quadric $Q$ of rank $\geq 5$ or

(ii) a Cohen-Macaulay divisor on a cone over the Veronese surface $W \subset \mathbb{P}^{5}$.

Then $X$ is arithmetically Cohen-Macaulay.

Proof. First suppose that $X$ satisfies (i). Then the claim is a consequence of Klein's theorem which can be shown as follows according to Hartshorne: The divisor class group of $Q$ is generated by the class of the hyperplane section of $Q$ (cf. [13], Ex. II.6.5). Therefore the homogeneous coordinate $\operatorname{ring} B$ of $Q$ is a factorial domain (cf. [13], Ex. II.6.3). Since $I(X) B$ is a prime ideal in $B$, it is a principal ideal $g B$ where $g$ is a form defining a hypersurface $G \subset \mathbb{P}^{n}$. Hence $X$ is the complete intersection $G \cap Q$.

In order to show the claim if $X$ satisfies (ii) we induct on the dimension of $X$. If $X$ is a curve on $W$, then $X$ is arithmetically Cohen-Macaulay since $W$ is the image of the second Veronese map of $\mathbb{P}^{2}$ in $\mathbb{P}^{5}$. Now let $X$ be a divisor on a cone $V$ over $W$ of dimension $>1$. Then the general hyperplane section $X \cap L$ of $X$ is a divisor on the general hyperplane section of $V$ which is again a cone over $W$. Hence $X \cap L$ is arithmetically Cohen-Macaulay by induction and the claim follows by Lemma 2.8 .

\section{Subschemes of a VARIETy of Minimal Degree}

If $X$ is an effective divisor on a variety $V$ of minimal degree, then this is also true for its general hyperplane section. In this section we want to show the converse of this statement under the assumption that $X$ is reduced and its degree is not too small. Finally we give a cohomological characterization of arithmetically Buchsbaum divisors on a variety of minimal degree which will serve as the starting point for the more precise results proved later on. 
We need some preparations. $X \subset \mathbb{P}^{n}$ will denote a subscheme of degree $d$ and codimension $c$ which is not even assumed to be equidimensional.

Lemma 3.1. Let $X$ be a subscheme contained in a variety $V$ of minimal degree and codimension $c-1$. If $X$ has degree $d>2 c$, then its homogeneous ideal is generated by quadrics and forms of degree $\geq\left\lceil\frac{d}{c}\right\rceil$. Moreover, the quadrics containing $X$ cut out $V$.

Proof. Let $F$ be a hypersurface of degree $s$ containing $X$ which does not contain $V$. Then $V \cap F$ is a subscheme of codimension $c$ and degree $s c$ containing $X$. It follows $d \leq s c$ proving the claim.

Let $M$ be an $R$-module. Its socle $\operatorname{soc}(M)$ is the set of elements of $M$ which are annihilated by the maximal ideal $\mathfrak{m}=\left(x_{0}, \ldots, x_{n}\right)$.

Lemma 3.2. Suppose that $X$ is a nondegenerate subscheme contained in a variety $V$ of minimal degree $c$. Let $f=\operatorname{depth} A$ where $A=R / I(X)$ and put $t=\left\lceil\frac{d}{c}\right\rceil \geq 2$. Then it holds

$$
a\left(\operatorname{soc}\left(H_{\mathfrak{m}}^{f}(A)\right)\right) \geq t-f-1 .
$$

Proof. Let

$$
0 \rightarrow F_{n-f+1} \stackrel{\varphi}{\longrightarrow} F_{n-f} \rightarrow \ldots \rightarrow F_{1} \rightarrow R \rightarrow A \rightarrow 0
$$

be a minimal free graded resolution of $A$ where $F_{i}=\bigoplus_{j} R\left(-e_{i, j}\right)$. First we want to show

$$
\min \left\{e_{n-f+1, j}\right\} \geq n-f+t .
$$

This is trivial if $t=2$ because by assumption $I(X)$ does not contain a form of degree one. Thus we may assume $t \geq 3$. Next, we note that $\left[\operatorname{Tor}_{i}^{R}(K, I(X) / I(V))\right]_{i+j}$ is the homology of the complex

$$
\bigwedge^{i+1}[R]_{1} \otimes[I(X) / I(V)]_{j-1} \rightarrow \bigwedge^{i}[R]_{1} \otimes[I(X) / I(V)]_{j} \rightarrow \bigwedge^{i-1}[R]_{1} \otimes[I(X) / I(V)]_{j+1} .
$$

Thus we obtain

$$
\left[\operatorname{Tor}_{i}^{R}(K, I(X) / I(V))\right]_{j}=0 \quad \text { if } j \leq i+t-1
$$

because we know by Lemma 3.1 that $[I(X) / I(V)]_{j}=0$ if $j<t$.

The exact sequence $0 \rightarrow I(V) \rightarrow I(X) \rightarrow I(X) / I(V) \rightarrow 0$ provides the following piece of a long exact sequence:

$$
\operatorname{Tor}_{c-1}^{R}(K, I(V)) \rightarrow \operatorname{Tor}_{c-1}^{R}(K, I(X)) \rightarrow \operatorname{Tor}_{c-1}^{R}(K, I(X) / I(V)) .
$$

Since $V$ is arithmetically Cohen-Macaulay, the resolution of $V$ has length $c-2$. Hence, the left-hand side of the exact sequence above vanishes. Therefore $(+)$ implies $\left[\operatorname{Tor}_{c}^{R}(K, A)\right]_{j} \cong\left[\operatorname{Tor}_{c-1}^{R}(K, I(X))\right]_{j}=0$ if $j \leq c+t-2$ which is equivalent to $\min \left\{e_{c, j}\right\} \geq c+t-1$. Since the resolution of $A$ above was chosen to be minimal, we get $\min \left\{e_{n-f+1, j}\right\} \geq n-f+t$ as desired.

Now, dualizing the resolution of $A$ above we obtain the exact sequence

$$
F_{n-f}^{*} \stackrel{\varphi^{*}}{\longrightarrow} F_{n-f+1}^{*} \rightarrow \operatorname{Ext}_{R}^{n-f+1}(A, R) \rightarrow 0 .
$$


This is the beginning of a minimal free resolution of $\operatorname{Ext}_{R}^{n-f+1}(A, R)$. Thus it follows that

$$
\operatorname{Ext}_{R}^{n-f+1}(A, R) \otimes_{R} K \cong F_{n-f+1}^{\prime} \otimes K \cong \bigoplus_{j} K\left(e_{n-f+1, j}\right) .
$$

Therefore we get the following chain of isomorphisms using duality:

$$
\begin{aligned}
\operatorname{soc}\left(H_{\mathfrak{m}}^{f}(A)\right) & \cong \operatorname{Hom}_{R}\left(K, H_{\mathfrak{m}}^{f}(A)\right) \\
& \cong \operatorname{Hom}_{R}\left(K, \operatorname{Hom}_{K}\left(\operatorname{Ext}_{R}^{n-f+1}(A, R), K\right)\right)(n+1) \\
& \cong \operatorname{Hom}_{K}\left(K \otimes_{R} \operatorname{Ext}_{R}^{n-f+1}(A, R), K\right)(n+1) \\
& \cong \operatorname{Hom}_{K}\left(\bigoplus_{j} K\left(e_{n-f+1, j}\right), K\right)(n+1) \\
& \cong \bigoplus_{j} K\left(-e_{n-f+1, j}+(n+1)\right) .
\end{aligned}
$$

It follows by $(*)$ that $a\left(\operatorname{soc}\left(H_{\mathfrak{m}}^{f}(A)\right)\right)=\min \left\{e_{n-f+1, j}\right\}-n-1 \geq t-f-1$.

Now we are ready for a more general version of the announced "lifting" result.

Proposition 3.3. Let $X \subset \mathbb{P}^{n}$ be a subscheme without zero-dimensional components. Suppose that $X$ has degree $>3 c$ and assume that $K$ has characteristic zero. Then $X$ is contained in a variety $V \subset \mathbb{P}^{n}$ of minimal degree $c$ if and only if its general hyperplane section $X \cap L$ is contained in a variety $W \subset L \cong \mathbb{P}^{n-1}$ of minimal degree $c$. In this case we have $W=V \cap L$.

Proof. It is clear that $X \cap L \subset V \cap L$ if $X \subset V$. Thus let us assume $Y=X \cap L \subset W$. Let $A=R / I(X)$ be the homogeneous coordinate ring of $X$ and let the hyperplane $L$ be defined by $l \in R$. If depth $A \geq 2$, then every minimal generator of $I(Y) \subset R / l R$ lifts to a minimal generator of $I(X)$ proving the claim.

Let depth $A=1$. The $\operatorname{ring} B=(A / l A) / H_{\mathfrak{m}}^{0}(A / l A)$ is the homogeneous coordinate ring of $Y \subset L$. The exact sequence $0 \rightarrow A(-1) \stackrel{l}{\longrightarrow} A \rightarrow A / l A \rightarrow 0$ induces a long exact cohomology sequence

$$
0 \rightarrow H_{\mathfrak{m}}^{0}(A / l A) \rightarrow H_{\mathfrak{m}}^{1}(A)(-1) \stackrel{l}{\longrightarrow} H_{\mathfrak{m}}^{1}(A) \underset{\substack{\searrow \\ \text { coker }}}{\longrightarrow} H_{\mathfrak{m}}^{1}(A / l A) \rightarrow \ldots
$$

Since $X$ has no zero-dimensional components, $H_{\mathfrak{m}}^{1}(A)$ is a finitely generated $R$ module and we may apply the Socle lemma of Huneke and Ulrich [14]. It gives

$$
a\left(H_{\mathfrak{m}}^{0}(A / l A)\right)>a(\operatorname{soc}(\operatorname{coker})) \geq a\left(\operatorname{soc}\left(H_{\mathfrak{m}}^{1}(A / l A)\right)\right) .
$$

Moreover, we have $H_{\mathfrak{m}}^{1}(A / l A) \cong H_{\mathfrak{m}}^{1}(B)$. Therefore we deduce with Lemma 3.2

$$
a\left(H_{\mathfrak{m}}^{0}(A / l A)\right) \geq t-1 \geq 3
$$

because $t=\left\lceil\frac{\operatorname{deg} X}{c}\right\rceil \geq 4$ by assumption. Since $H_{\mathfrak{m}}^{0}(A / l A) \cong I(Y) /((I(X)+l R) / l R)$, we conclude that the quadrics containing $Y$ lift to quadrics containing $X$. By Lemma 3.1 the intersection of the quadrics containing $Y$ is $W$. Therefore the quadrics containing $X$ cut out a subscheme $V$ with $V \cap L=W$. Hence, $V$ must be a variety of minimal degree. 
The assumption that the characteristic of the field $K$ is zero was only necessary in order to allow the application of the Socle lemma of [14] in the proof above. We do not need this if $X$ is arithmetically Buchsbaum.

Lemma 3.4. If we drop in Proposition 3.3 the assumption that $K$ has characteristic zero but assume in addition that $X$ is arithmetically Buchsbaum, then the claim of Proposition 3.3 is still true.

Proof. We use the notation of the previous proof. If $X$ is arithmetically Buchsbaum, then $H_{\mathfrak{m}}^{0}(A / l A) \cong H_{\mathfrak{m}}^{1}(A)(-1) \cong \operatorname{coker}(-1) \cong \operatorname{soc}(\operatorname{coker}(-1))$. It follows $a\left(H_{\mathfrak{m}}^{0}(A / l A)\right)>a(\operatorname{soc}($ coker $))$ and now we can conclude as in the proof of Proposition 3.3 .

Now we want to analyze the cohomology of arithmetically Buchsbaum divisors on a variety of minimal degree. At first we consider curves.

Lemma 3.5. Let $X \subset \mathbb{P}^{n}$ be a reduced arithmetically Buchsbaum curve lying on a surface of minimal degree. Let $d=\operatorname{deg} X=t c+1-p$ where $1 \leq p \leq c=n-1$. Suppose $t \geq 2$ and that $X$ is connected if $t=2$. Then it holds

$$
h^{1}\left(\mathcal{J}_{X}(j)\right) \leq \begin{cases}\min \{p, c-p\} & \text { if } j=t-1 \\ 0 & \text { otherwise }\end{cases}
$$

Proof. Let $A$ be the homogeneous coordinate ring of $X$. Let $l$ be a general linear form defining the hyperplane $L \subset \mathbb{P}^{n}$. Since $A$ is Buchsbaum, we have an exact sequence

$$
0 \rightarrow H_{\mathfrak{m}}^{0}(A / l A) \rightarrow H_{\mathfrak{m}}^{1}(A)(-1) \stackrel{l}{\longrightarrow} H_{\mathfrak{m}}^{1}(A) \rightarrow H_{\mathfrak{m}}^{1}(A / l A) \rightarrow \ldots
$$

where the indicated multiplication map vanishes. Therefore we obtain $H_{\mathfrak{m}}^{0}(A / l A) \cong$ $H_{\mathfrak{m}}^{1}(A)(-1)$ and an embedding $H_{\mathfrak{m}}^{1}(A) \hookrightarrow H_{\mathfrak{m}}^{1}(A / l A)$.

Moreover, $Y=X \cap L$ is a set of $d$ points on a rational normal curve $C$. It follows for $j \geq 0$

$$
\operatorname{rank}\left[H_{\mathfrak{m}}^{1}(A / l A)\right]_{j}=d-h_{Y}(j)=d-\min \{d, j c+1\} .
$$

In particular, we get $e\left(H_{\mathfrak{m}}^{1}(A / l A)\right) \leq t-1$ and $\operatorname{rank}\left[H_{\mathfrak{m}}^{1}(A / l A)\right]_{t-1}=c-p$. This implies

$$
e\left(H_{\mathfrak{m}}^{1}(A)\right) \leq t-1 \quad \text { and } \quad \operatorname{rank}\left[H_{\mathfrak{m}}^{1}(A)\right]_{t-1} \leq c-p .
$$

Since $A$ is Buchsbaum, we have

$$
H_{\mathfrak{m}}^{0}(A / l A)(1) \cong H_{\mathfrak{m}}^{1}(A) \cong \operatorname{soc}\left(H_{\mathfrak{m}}^{1}(A)\right) \hookrightarrow \operatorname{soc}\left(H_{\mathfrak{m}}^{1}(A / l A)\right) .
$$

Therefore Lemma 3.2 applied to $Y$ provides $t-1 \leq a\left(H_{\mathfrak{m}}^{0}(A / l A)\right)$. We want to show that even $t \leq a\left(H_{\mathfrak{m}}^{0}(A / l A)\right)=a\left(H_{\mathfrak{m}}^{1}(A)\right)+1$ holds.

We have $H_{\mathfrak{m}}^{0}(A / l A) \cong I(Y) /((I(X)+l R) / l R)$. Let $t \geq 4$. Then $I(Y)$ has minimal generators only in degree 2 and $t$ due to Proposition 2.2. It follows $t \leq$ $a\left(H_{\mathfrak{m}}^{0}(A / l A)\right)$. If $t=2$, then the connectedness of $X$ implies $\left[H_{\mathfrak{m}}^{1}(A)\right]_{0}=0$; thus $a\left(H_{\mathfrak{m}}^{1}(A)\right) \geq 1$. Let $t=3$, i.e. $2 c+1 \leq d \leq 3 c$. Suppose $[I(Y) /((I(X)+l R) / l R)]_{2} \neq$ 0 . It follows that $I(Y)$ contains a quadric which defines a quadric hypersurface $Q$ not containing the curve $C$. Therefore $C \cap Q$ is a zero-dimensional scheme containing $Y$. Thus we get $\operatorname{deg} Y \leq \operatorname{deg} C \cap Q=2 c$ which contradicts $t=3$. Therefore we have seen that in any case

$$
a\left(H_{\mathfrak{m}}^{1}(A)\right) \geq t-1
$$


Using the graded isomorphism $H_{\mathfrak{m}}^{1}(A) \cong H_{*}^{1}\left(\mathcal{J}_{X}\right)$ it only remains to show that $h^{1}\left(\mathcal{J}_{X}(t-1)\right) \leq p$. Consider again the isomorphisms

$$
H_{\mathfrak{m}}^{1}(A)(-1) \cong H_{\mathfrak{m}}^{0}(A / l A) \cong I(Y) /((I(X)+l R) / l R) .
$$

We know that this module is concentrated in degree $t$. But according to Theorem 2.4 $I(Y)$ only contains $p$ minimal generators in degree $t$ besides the minimal generators of $I(C)$. This completes the proof.

The next result shows that the cohomology of effective arithmetically Buchsbaum divisors on a variety of minimal degree is particularly simple. It will be strengthened later on but is needed as an ingredient to achieve this improvement.

Proposition 3.6. Let $X \subset \mathbb{P}^{n}$ be a reduced positive-dimensional subscheme where $t=\left\lceil\frac{d}{c}\right\rceil \geq 2$. Suppose $X$ is a divisor on a variety of minimal degree. Then $X$ is arithmetically Buchsbaum if and only if $H^{i}\left(\mathcal{J}_{X}(j)\right)=0$ if $j \neq t-i$ and $1 \leq i \leq \operatorname{dim} X$.

Proof. Let $A=R / I(X)$ be the homogeneous coordinate ring of $X$. Then the isomorphisms $H_{\mathfrak{m}}^{i}(A) \cong H_{*}^{i}\left(\mathcal{J}_{X}\right)(1 \leq i<n)$ and Proposition I.3.10 in [24] show that the cohomological conditions above imply the Buchsbaum property of $X$.

In order to show the converse we induct on the dimension $k$ of $X$. If $X$ is a curve, the claim follows by Lemma 3.5. Let $k>1$. Consider a general hyperplane $L \subset \mathbb{P}^{n}$ defined by $l \in R$. Let $B$ be the homogeneous coordinate ring of $Y=X \cap L$. There is an exact sequence

$$
0 \rightarrow H_{\mathfrak{m}}^{0}(A / l A) \rightarrow A / l A \rightarrow B \rightarrow 0 .
$$

Due to the finite length of $H_{\mathfrak{m}}^{0}(A / l A)$ the long exact cohomology sequence provides

$$
H_{\mathfrak{m}}^{i}(A / l A) \cong H_{\mathfrak{m}}^{i}(B) \quad \text { if } i \geq 1 .
$$

Since $Y$ is arithmetically Buchsbaum, too, the induction hypothesis gives

$$
\left[H_{\mathfrak{m}}^{i}(A / l A)\right]_{j}=0 \quad \text { if } j \neq t-i \text { and } 1 \leq i<k .
$$

According to the Buchsbaum property of $X$ we know that $l$ annihilates $H_{\mathfrak{m}}^{i}(A)$ if $1 \leq i \leq k$. Therefore the long exact cohomology sequence induced by

$$
0 \rightarrow A(-1) \stackrel{l}{\longrightarrow} A \rightarrow A / l A \rightarrow 0
$$

splits into short exact sequences

$$
0 \rightarrow H_{\mathfrak{m}}^{i}(A) \rightarrow H_{\mathfrak{m}}^{i}(A / l A) \rightarrow H_{\mathfrak{m}}^{i+1}(A)(-1) \rightarrow 0, \quad 1 \leq i<k .
$$

Therefore $(*)$ proves the claim.

\section{Castelnuovo bounds}

The goal of this section is to characterize the arithmetically Buchsbaum varieties which achieve the regularity bound of Stückrad and Vogel [23].

The first observation shows that it is useful to consider hyperplane sections in order to derive bounds for the regularity of an arithmetically Buchsbaum scheme. It follows from [23], Lemma 2 (cf. also [20], Proposition 4.2).

Lemma 4.1. Let $X \subset \mathbb{P}^{n}$ be an arithmetically Buchsbaum subscheme of positive dimension. Let $L$ be a general hyperplane of $\mathbb{P}^{n}$. Then it holds

$$
\operatorname{reg} X=\operatorname{reg} X \cap L \text {. }
$$


We wish to use this result in the following way: Let $L_{c} \subset \mathbb{P}^{n}$ be a general linear subspace of dimension $c=\operatorname{codim} X$. If $X$ is reduced, then $Y=X \cap L_{c}$ is a set of $d$ points in $L_{c} \cong \mathbb{P}^{c}$ and the lemma above implies $\operatorname{reg} X=\operatorname{reg} Y$.

Moreover, the Castelnuovo-Mumford regularity of $Y$ is the smallest integer $j$ such that $h_{Y}(j-1)=\operatorname{deg} Y$ and $h_{Y}(j-2)<\operatorname{deg} Y$ where $h_{Y}$ denotes the Hilbert function of $Y$ (cf., for example, [17], Remark 2.3). Hence, lower bounds for the Hilbert function of $Y$ imply upper bounds for the regularity of $X$.

We keep the notation just introduced throughout the rest of this section. A first application of the strategy above yields:

Lemma 4.2. Let $X \subset \mathbb{P}^{n}$ be an effective reduced arithmetically Buchsbaum divisor on a variety of minimal degree $c$. Then

$$
\operatorname{reg} X=\left\lceil\frac{d-1}{c}\right\rceil+1
$$

Moreover, if $d>c$ and $c$ divides $d-1$, then $X$ is arithmetically Cohen-Macaulay.

Proof. Since $X$ is a divisor on a variety of minimal degree, $Y$ is a divisor on a rational normal curve of $L_{c} \cong \mathbb{P}^{c}$. Hence, the Hilbert function of $Y$ for $j \geq 0$ is

$$
h_{Y}(j)=\min \{d, j c+1\} .
$$

It follows reg $X=\operatorname{reg} Y=\left\lceil\frac{d-1}{c}\right\rceil+1$ proving the first claim.

Now suppose that $c$ divides $d-1$. Then $t=\left\lceil\frac{d}{c}\right\rceil=\left\lceil\frac{d-1}{c}\right\rceil+1$ and from the first claim we read off $e\left(H_{*}^{i}\left(\mathcal{J}_{X}\right)\right) \leq t-1-i$ for all $i$. Therefore Proposition 3.6 implies $H_{*}^{i}\left(\mathcal{J}_{X}\right)=0$ if $1 \leq i \leq \operatorname{dim} X$, i.e., $X$ is arithmetically Cohen-Macaulay.

Proposition 4.3. Let $X \subset \mathbb{P}^{n}$ be an integral arithmetically Buchsbaum scheme. Then it holds:

(a)

$$
\operatorname{reg} X \leq\left\lceil\frac{d-1}{c}\right\rceil+1
$$

(b) Suppose in addition $c \geq 3$ and

(i) $\operatorname{char}(K)=0, d \geq \min \{3 c+1,2 c+5\}$ or

(ii) $c \geq 5, d \geq \min \{6 c-7,25\}$.

If $X$ is not a divisor on a variety of minimal degree, then

$$
\operatorname{reg} X \leq\left\lceil\frac{d}{c+1}\right\rceil+1+\mu
$$

$$
\text { where } \mu= \begin{cases}1 & \text { if } c+1 \text { divides } d \\ 0 & \text { otherwise. }\end{cases}
$$

Proof. Applying the strategy described above we have to look at the Hilbert function of $Y$. According to [1], Theorem 0.1 it satisfies for $j \geq 0$

$$
h_{Y}(j) \geq \min \{d, j c+1\} .
$$

This implies the first claim.

The second claim is a consequence of the following estimation if $j \geq 0$ :

$$
h_{Y}(j) \geq \begin{cases}\min \{d, j(c+1)\} & \text { if } \quad j \neq \frac{d}{c+1}-1 \\ d-1 & \text { if } \quad j=\frac{d}{c+1}-1 .\end{cases}
$$


In order to see this note that $Y$ does not lie on a rational normal curve due to Lemma 3.4. Thus the inequality above follows as in the proof of [17], Proposition 4.2 because in case of $\operatorname{char}(K)=0$ the Uniform position lemma in [10] and [12], Proposition 3.20 apply.

Claim (a) of the proposition above under the additional assumption $\operatorname{char}(K)=0$ is one of the main results of [23]. Claim (b) is new.

In the second claim of the statement above the case $c=2$ was excluded because then we have a weaker bound only.

Lemma 4.4. Let $X \subset \mathbb{P}^{n}$ be an integral arithmetically Buchsbaum scheme of codimension 2 which is not a divisor on a variety of minimal degree. Suppose that the field $K$ has characteristic zero. Then

$$
\operatorname{reg} X \leq\left\lceil\frac{2 d-1}{5}\right\rceil+1
$$

In particular, $\operatorname{reg} X \leq\left\lceil\frac{d-1}{2}\right\rceil$ if $d \geq 10$ and $\operatorname{reg} X<\left\lceil\frac{d}{2}\right\rceil$ if $d \geq 15$.

Proof. As before we get by Lemma 3.4 that $Y \subset \mathbb{P}^{2}$ is not contained in a rational normal curve, that is, $Y$ is not contained in a quadric. Thus $h_{Y}(2)=6$. According to Harris's Uniform position lemma [10] $Y$ is in uniform position. Therefore [12], Corollary 3.5 implies

$$
h_{Y}(j) \geq \begin{cases}\min \left\{d, \frac{5}{2} j+1\right\} & \text { if } j \text { is even, } \\ \min \left\{d, \frac{5}{2}(j-1)+3\right\} & \text { if } j \text { is odd. }\end{cases}
$$

The assertions follow.

Now it is easy to characterize the extremal varieties.

Theorem 4.5. Let $X \subset \mathbb{P}^{n}$ be an integral arithmetically Buchsbaum subscheme of degree $d>\max \{2 c(c+1), 15\}$. Suppose either char $(K)=0$ or $c \geq 5$. Then the following conditions are equivalent:

(a) $\operatorname{reg} X=\left\lceil\frac{d-1}{c}\right\rceil+1$.

(b) $m(X) \geq\left\lceil\frac{d}{c}\right\rceil$.

(c) $X$ is a divisor on a variety of minimal degree.

Proof. The assumption on the degree $d$ of $X$ ensures that we may apply Proposition 4.3 and Lemma 4.4. Since

$$
\left\lceil\frac{d}{c+1}\right\rceil+1+\mu<\left\lceil\frac{d}{c}\right\rceil \text { if } d>2 c(c+1)
$$

these results show that (a) as well as (b) implies (c). The converse is furnished by Lemma 4.2 and Lemma 3.1.

Remark 4.6. (i) The equivalence of (a) and (c) in the theorem above holds already true under the slightly weaker hypothesis $d>(c+1)^{2}$. This follows from the estimate

$$
\left\lceil\frac{d}{c+1}\right\rceil+\mu<\left\lceil\frac{d-1}{c}\right\rceil \text { if } d>(c+1)^{2} .
$$


(ii) By inspection of

$$
\left\lceil\frac{d}{c}\right\rceil \leq m(X) \leq \operatorname{reg} X=\left\lceil\frac{d-1}{c}\right\rceil+1
$$

we may conclude that our last theorem allows at most two possibilities for $m(X)$. If we compare the statement with Theorem 2.1, the question arises if it is possible to replace condition (b) in Theorem 4.5 by $m(X)=\left\lceil\frac{d}{c}\right\rceil$. An answer will be given in the next section. It requires a detailed study of the varieties to which Theorem 4.5 applies.

\section{Arithmetically Buchsbaum divisors on rational normal scrolls}

The aim of this section is to derive a characterization of reduced arithmetically Buchsbaum subschemes which are divisors on a rational normal scroll. We also establish an existence result for these effective divisors and compute their Hilbert function, cohomology and often all graded Betti numbers.

First we give a description of scrolls following [5] and [11]. Let $\mathcal{E}=\bigoplus_{i=0}^{k} \mathcal{O}_{\mathbb{P}^{1}}\left(a_{i}\right)$ be a vector bundle on $\mathbb{P}^{1}$ where $0 \leq a_{0} \leq \ldots \leq a_{k}$ and $a_{k}>0 . \mathcal{E}$ is generated by $k+1+\sum a_{i}$ global sections. Write $\mathbb{P}(\mathcal{E})$ for the projectivized vector bundle

$$
\mathbb{P}(\mathcal{E})=\operatorname{Proj} \operatorname{Sym} \mathcal{E} \stackrel{\pi}{\longrightarrow} \mathbb{P}^{1}
$$

and let $\mathcal{O}_{\mathbb{P}(\mathcal{E})}(1)$ be the tautological line bundle. Then $\mathcal{O}_{\mathbb{P}(\mathcal{E})}(1)$ is generated by its global sections and defines a birational map

$$
\mathbb{P}(\mathcal{E}) \longrightarrow \mathbb{P}^{k+\sum a_{i}}
$$

We write $S(\mathcal{E})$ or $S\left(a_{0}, \ldots, a_{k}\right)$ for the image of this map. It is a variety of dimension $k+1$ and minimal degree $c=\sum a_{i}$. A rational normal scroll is one of the varieties $S(\mathcal{E})$. The divisor class group of $S=S(\mathcal{E})$ is generated by the hyperplane section $H$ and a linear subspace $F \subset S$ of dimension $k$.

We will use the notation just introduced throughout the whole section. We need several technical results.

Lemma 5.1. (a) The canonical class of $S$ is

$$
K_{S} \sim-(k+1) H+(c-2) F .
$$

(b) For any integers $a, b$ we have

$h^{0}\left(S, \mathcal{O}_{S}(a H+b F)\right)=\left\{\begin{array}{l}0 \quad \text { if } a<0, \\ \sum_{j_{0}+\ldots+j_{k}=a} \max \left\{0, j_{0} a_{0}+\ldots+j_{k} a_{k}+b+1\right\} \quad \text { if } a \geq 0 .\end{array}\right.$

(c) If $a$ and $b$ are non-negative then

$$
h^{0}\left(S, \mathcal{O}_{S}(a H+b F)\right)=c\left(\begin{array}{c}
a+k \\
1+k
\end{array}\right)+(b+1)\left(\begin{array}{c}
a+k \\
k
\end{array}\right) .
$$

Proof. Claim (a) is the lemma on page 55 in [11].

According to [11] or [6]

$$
h^{0}\left(S, \mathcal{O}_{S}(a H+b F)\right)=h^{0}\left(\mathbb{P}^{1}, \operatorname{Sym}_{a}(\mathcal{E}) \otimes \mathcal{O}_{\mathbb{P}^{1}}(b)\right)
$$

which proves (b). 
Now suppose that $a \geq 0$ and $b \geq 0$. Then

$$
h^{0}\left(S, \mathcal{O}_{S}(a H+b F)\right)=\sum_{\substack{j_{0}+\ldots+j_{k}=a \\ j_{i} \geq 0}}\left(j_{0} a_{0}+\ldots+j_{k} a_{k}+b+1\right) .
$$

Denote by $n_{k+1}(t)$ the number of $(k+1)$-tuples $\left(j_{0}, \ldots, j_{k}\right)$ of non-negative integers with $j_{0}+\ldots+j_{k}=t$. Then it is easy to see by induction on $k$ that $n_{k+1}(t)=\left(\begin{array}{c}t+k \\ k\end{array}\right)$. Moreover, we observe that the sum above does not change if we replace an $a_{i}>0$ by $a_{i}-1$ and an $a_{j}(j \neq i)$ by $a_{j}+1$. Using $c=a_{0}+\ldots+a_{k}$ it follows

$$
\begin{aligned}
h^{0}\left(S, \mathcal{O}_{S}(a H+b F)\right) & =n_{k+1}(a)(b+1)+\sum_{j_{0}+\ldots+j_{k}=a} j_{0} c \\
& =(b+1)\left(\begin{array}{c}
a+k \\
k
\end{array}\right)+\sum_{j_{0}=0}^{a} j_{0} c n_{k}\left(a-j_{0}\right) \\
& =(b+1)\left(\begin{array}{c}
a+k \\
k
\end{array}\right)+c \sum_{j=0}^{a}(a-j)\left(\begin{array}{c}
j+k-1 \\
j
\end{array}\right) \\
& =(b+1)\left(\begin{array}{c}
a+k \\
k
\end{array}\right)+c\left(\begin{array}{c}
a+k \\
1+k
\end{array}\right)
\end{aligned}
$$

where the formula needed to prove the last equality is easily shown by induction on $a$.

The third claim of the lemma above shows in particular that the cohomology of effective divisors on $S=S\left(a_{0}, \ldots, a_{k}\right)$ depends only on its class and the degree and dimension of $S$ and does not depend on the specific $a_{i}$ 's.

For later purpose we recall the following result of Serre [22].

Lemma 5.2. Let $Z \subset \mathbb{P}^{n}$ be a projective subscheme. Then it holds for all integers $j$

$$
h_{Z}(j)-p_{Z}(j)=\sum_{i=1}^{\operatorname{dim} Z+1}(-1)^{i} h^{i}\left(\mathcal{J}_{Z}(j)\right) .
$$

Next we want to use Proposition 3.6 in order to derive a characterization of arithmetically Buchsbaum divisors which allows induction on the dimension.

Lemma 5.3. Let $X \subset \mathbb{P}^{n}$ be an effective reduced divisor on a variety of minimal degree. Suppose $k=\operatorname{dim} X \geq 1$ and $d=\operatorname{deg} X>c=n-k$. Then $X$ is arithmetically Buchsbaum if and only if

(a) $X$ is (locally) Cohen-Macaulay and

(b) the general hyperplane section of $X$ is arithmetically Buchsbaum and

(c) $h_{X}(j)-p_{X}(j)=(-1)^{k+1} h^{k+1}\left(\mathcal{J}_{X}(j)\right)$ if $j=t$ and if $j=t-k-1$ where $t=\left\lceil\frac{d}{c}\right\rceil$.

Proof. Suppose $X$ is arithmetically Buchsbaum. Then it is well-known that $X$ satisfies (a) and (b). It remains to show (c). Due to Lemma 5.2 we have

$$
h_{X}(j)-p_{X}(j)+(-1)^{k} h^{k+1}\left(\mathcal{J}_{X}(j)\right)=\sum_{i=1}^{k}(-1)^{i} h^{i}\left(\mathcal{J}_{X}(j)\right) .
$$


According to Proposition 3.6 we already know that $H_{*}^{i}\left(\mathcal{J}_{X}\right)$ is concentrated in degree $t-i$ if $1 \leq i \leq k$. Hence, the right-hand side of the equality above vanishes if $j=t$ or $j=t-k-1$ proving (c).

In order to show the sufficiency of the conditions (a)-(c) consider a general hyperplane $L$. We have exact sequences

$$
H^{i}\left(\mathcal{J}_{X}(j-1)\right) \rightarrow H^{i}\left(\mathcal{J}_{X}(j)\right) \rightarrow H^{i}\left(\mathcal{J}_{X \cap L}(j)\right) \quad(i \geq 1, j \in \mathbb{Z}) .
$$

Due to (b) we may apply Proposition 3.6 and obtain that $H_{*}^{i}\left(\mathcal{J}_{X \cap L}\right)$ is concentrated in degree $t-i$ if $1 \leq i \leq k-1$. Since $X$ is Cohen-Macaulay, we get that $H^{i}\left(\mathcal{J}_{X}(j)\right)=$ 0 for all $j \ll 0$ if $i \leq k$. Therefore the exact sequences above imply

$$
a\left(H_{*}^{i}\left(\mathcal{J}_{X}\right)\right) \geq a\left(H_{*}^{i}\left(\mathcal{J}_{X \cap L}\right)\right) \geq t-i \quad \text { if } 1 \leq i<k .
$$

Hence, we obtain by condition (c) and Lemma 5.2

$$
\begin{aligned}
0 & =h_{X}(t-k-1)-p_{X}(t-k-1)+(-1)^{k} h^{k+1}\left(\mathcal{J}_{X}(t-k-1)\right) \\
& =(-1)^{k} h^{k}\left(\mathcal{J}_{X}(t-k-1)\right) .
\end{aligned}
$$

That's why the exact sequences

$$
0 \rightarrow H^{k-1}\left(\mathcal{J}_{X \cap L}(j)\right) \rightarrow H^{k}\left(\mathcal{J}_{X}(j-1)\right) \rightarrow H^{k}\left(\mathcal{J}_{X}(j)\right)
$$

imply $a\left(H_{*}^{k}\left(\mathcal{J}_{X}\right)\right) \geq t-k$ because $a\left(H_{*}^{k-1}\left(\mathcal{J}_{X \cap L}\right)\right) \geq t-k+1$ by Proposition 3.6.

Thus we have shown that $a\left(H_{*}^{i}\left(\mathcal{J}_{X}\right)\right) \geq t-i$ if $1 \leq i \leq k$. On the other hand Lemma 4.2 provides $e\left(H_{*}^{i}\left(\mathcal{J}_{X}\right)\right)<e\left(H_{*}^{i-1}\left(\mathcal{J}_{X \cap L}\right)\right) \leq t-i+1$ for all $i \geq 2$.

This implies that $H_{*}^{i}\left(\mathcal{J}_{X}\right)$ is concentrated in degree $t-i$ if $2 \leq i \leq k$ and $e\left(H_{*}^{k+1}\left(\mathcal{J}_{X}\right)\right) \leq t-k+1$. Using condition (c) with $j=t$ it follows $H^{1}\left(\mathcal{J}_{X}(t)\right)=0$. Hence $X$ is $t$-regular and we get that also $H_{*}^{1}\left(\mathcal{J}_{X}\right)$ is concentrated in degree $t-1$. Therefore Proposition 3.6 shows that $X$ is arithmetically Buchsbaum.

In order to derive necessary conditions for the property of being an arithmetically Buchsbaum divisor on a rational normal scroll we need some more preparations.

Lemma 5.4. Let $V \subset \mathbb{P}^{n}$ be a variety of minimal degree $c$. Let $k+1=n+1-c$ denote the dimension of $V$. Then

(a) $p_{V}(j)=c\left(\begin{array}{c}k+j \\ k+1\end{array}\right)+\left(\begin{array}{c}k+j \\ k\end{array}\right)$ for all $j \in \mathbb{Z}$;

(b) $h_{V}(j)= \begin{cases}0 & \text { if } j<0 \\ p_{V}(j) & \text { if } j \geq-k+1\end{cases}$

(c) $\left.h^{k+2}\left(\mathcal{J}_{V}\right)\right)= \begin{cases}0 & \text { if } j \geq-k+1 \\ (-1)^{k+1} p_{V}(j) & \text { if } j<0\end{cases}$

In particular, we have $e\left(H_{*}^{k+2}\left(\mathcal{J}_{V}\right)\right)=-k$.

Proof. Claims (a) and (b) are probably well-known. In any case they follow easily by induction on $k$ using the fact that $V$ is arithmetically Cohen-Macaulay. This property also implies by Lemma $5.2 h_{V}(j)-p_{V}(j)=(-1)^{k} h^{k+2}\left(\mathcal{J}_{V}(j)\right)$ proving (c).

From now on we will always assume that $S \subset \mathbb{P}^{n}$ is a rational normal scroll of dimension $k+1$ where $2 \leq k+1<n$.

Lemma 5.5. Let $X \subset \mathbb{P}^{n}$ be a reduced Cohen-Macaulay subscheme of codimension $c$ being a divisor on a rational normal scroll $S$. Then 
(a) $h_{X}(j)=h_{S}(j)-h^{0}\left(S, \mathcal{O}_{S}(-X+j H)\right) \quad$ for all $j \in \mathbb{Z}$,

(b) $p_{X}(j)=p_{S}(j)+(-1)^{k} h^{k+1}\left(S, \mathcal{O}_{S}(-X+j H)\right) \quad$ for all $j \ll 0$,

(c) $h^{k+1}\left(\mathcal{J}_{X}(j)\right)=h^{k+1}\left(S, \mathcal{O}_{S}(-X+j H)\right)-h^{k+2}\left(\mathcal{J}_{S}(j)\right) \quad$ for all $j \in \mathbb{Z}$.

Proof. Consider the exact sequence

$$
0 \rightarrow \mathcal{J}_{S}(j) \rightarrow \mathcal{J}_{X}(j) \rightarrow \mathcal{O}_{S}(-X+j H) \rightarrow 0 .
$$

Since $S$ is arithmetically Cohen-Macaulay, the induced long exact cohomology sequence yields exact sequences

$$
0 \rightarrow H^{0}\left(\mathcal{J}_{S}(j)\right) \rightarrow H^{0}\left(\mathcal{J}_{X}(j)\right) \rightarrow H^{0}\left(S, \mathcal{O}_{S}(-X+j H)\right) \rightarrow 0
$$

and

$$
0 \rightarrow H^{k+1}\left(\mathcal{J}_{X}(j)\right) \rightarrow H^{k+1}\left(S, \mathcal{O}_{S}(-X+j H)\right) \rightarrow H^{k+2}\left(\mathcal{J}_{S}(j)\right) \rightarrow 0
$$

proving (a) and (c).

The assumptions on $X$ imply $h^{i}\left(\mathcal{J}_{X}(j)\right)=0$ for all $j \ll 0$ if $0<i \leq k$. Hence, Lemma 5.2 provides

$$
-p_{X}(j)=(-1)^{k+1} h^{k+1}\left(\mathcal{J}_{X}(j)\right) \text { for all } j \ll 0 .
$$

Thus, claim (b) follows using (c) and Lemma 5.4, (c).

Suppose for the time being that $X$ is a curve. Then we denote by $g_{X}$ its arithmetic genus. We define

$$
\pi(d, n)=\frac{t(t-1)}{2} c-p(t-1)
$$

where as before $d=t c+1-p$ with $1 \leq p \leq c=n-1$. Note that $\pi(d, n)$ is the maximal genus of an integral curve in $\mathbb{P}^{n}$ of degree $d$ (cf., for example, [12]).

The next result sorts out the divisor classes on a rational normal scroll which we have to study more carefully.

Lemma 5.6. Let $X \subset \mathbb{P}^{n}$ be a reduced curve lying on a rational normal surface. Let $X \sim \alpha H+\beta F$. If $X$ is arithmetically Buchsbaum, then

$$
\begin{aligned}
& \alpha=t+1 \text { and } 1-\frac{3}{2} c \leq \beta \leq-c \quad \text { or } \\
& \alpha=t \text { and } 1-c \leq \beta \leq 0 \text { or } \\
& \alpha=t-1 \text { and } 1 \leq \beta \leq \frac{c}{2}+1 \quad \text { where } t=\left\lceil\frac{d}{c}\right\rceil .
\end{aligned}
$$

Moreover, $X$ is arithmetically Cohen-Macaulay if and only if $X \sim t H+\beta F$ where $1-c \leq \beta \leq 0$ or $X \sim(t-1) H+F$.

Proof. (I) We claim: If $X$ is arithmetically Buchsbaum, then

$$
\pi(d, n)-g_{X}=\operatorname{rank} H_{*}^{1}\left(\mathcal{J}_{X}\right) .
$$

In order to see this we note that the general hyperplane section $Z$ of $X$ lies on a rational normal curve. It follows $\pi(d, n)=\sum_{j>1}\left(d-h_{Z}(j)\right)$. Hence, the claim is a consequence of the equality $(*)$ in the proof of Theorem 6 in [21].

(II) Next, we want to show that the arithmetic genus of $X$ is

$$
g_{X}=\frac{\alpha(\alpha-1)}{2} c+(\alpha-1)(\beta-1) .
$$


We distinguish two cases. First assume $\beta \leq 0$. Then we get by Lemma 5.1

$$
\begin{aligned}
h^{0}\left(S, \mathcal{O}_{S}(-X+j H)\right) & =h^{0}\left(S, \mathcal{O}_{S}((j-\alpha) H-\beta F)\right) \\
& =c\left(\begin{array}{c}
j-\alpha+1 \\
2
\end{array}\right)+(1-\beta)\left(\begin{array}{c}
j-\alpha+1 \\
1
\end{array}\right) \quad \text { if } j \geq \alpha .
\end{aligned}
$$

Since the last expression is a polynomial in $j$, Lemma 5.5,(a) implies

$$
\begin{aligned}
p_{X}(j) & =p_{S}(j)-c\left(\begin{array}{c}
j-\alpha+1 \\
2
\end{array}\right)-(1-\beta)(j-\alpha+1) \\
& =(\alpha c+\beta) j+1-\left[\frac{\alpha(\alpha-1)}{2} c+(\alpha-1)(\beta-1)\right]
\end{aligned}
$$

proving the claim.

Suppose now $\beta>0$. Then Serre duality and Lemma 5.1 provide

$$
\begin{aligned}
h^{2}\left(S, \mathcal{O}_{S}(-X+j H)\right) & =h^{0}\left(S, \mathcal{O}_{S}\left(K_{S}+X-j H\right)\right) \\
& =h^{0}\left(S, \mathcal{O}_{S}((\alpha-2-j) H+(\beta+c-2) F)\right) \\
& =c\left(\begin{array}{c}
\alpha-1-j \\
2
\end{array}\right)+(\beta+c-1)\left(\begin{array}{c}
\alpha-1-j \\
1
\end{array}\right) \quad \text { if } j \leq \alpha-2 .
\end{aligned}
$$

The last expression is a polynomial in $j$. Hence Lemma 5.5,(b) gives

$$
\begin{aligned}
p_{X}(j) & =p_{S}(j)-c\left(\begin{array}{c}
\alpha-j-1 \\
2
\end{array}\right)-(\beta+c-1)(\alpha-j-1) \\
& =(\alpha c+\beta) j+1-\left[\frac{\alpha(\alpha-1)}{2} c+(\alpha-1)(\beta-1)\right]
\end{aligned}
$$

completing step (II).

(III) Since $d=t c+1-p=\alpha c+\beta$, we may write $\alpha=t-s$ and $\beta=s c+1-p$ for some integer $s$. Then we get

$$
\pi(d, n)-g_{X}=s\left(\frac{s+1}{2} c-p\right) .
$$

According to step (I) and Proposition 3.6 this implies

$$
s\left(\frac{s+1}{2} c-p\right)=\operatorname{rank} H_{*}^{1}\left(\mathcal{J}_{X}\right)=h^{1}\left(\mathcal{J}_{X}(t-1)\right) .
$$

It follows: If $s<-1$, then $h^{1}\left(\mathcal{J}_{X}(t-1)\right)>p$ contradicting Lemma 3.5. If $s>1$, then $h^{1}\left(\mathcal{J}_{X}(t-1)\right)>c-p$ contradicting Lemma 3.5 , too. If $s=-1$, then Lemma 3.5 yields $p \leq \min \{p, c-p\}$; thus $1 \leq p \leq \frac{c}{2}$. If $s=1$, then Lemma 3.5 yields $c-p \leq \min \{p, c-p\}$; thus $\frac{c}{2} \leq p \leq c$. Moreover, we see that $H_{*}^{1}\left(\mathcal{J}_{X}\right)=0$ if and only if $s=0$ or $s=1$ and $p=c$ completing the proof.

Remark 5.7. If $X$ and $S$ are smooth, then the claim of step (II) follows also by the Adjunction formula.

According to Lemma 5.6 and Lemma 5.3 we have two possibilities for finding arithmetically Buchsbaum divisors on a scroll which are not arithmetically CohenMacaulay. We treat them separately.

Lemma 5.8. Let $X \subset \mathbb{P}^{n}$ be a reduced Cohen-Macaulay subscheme being a divisor on a rational normal scroll $S=S\left(a_{0}, \ldots, a_{k}\right)$. Suppose $d>c$ and $X \sim(t-1) H+$ $(1+c-p) F$. Then $X$ is arithmetically Buchsbaum if and only if $a_{i} \geq c-p$ for all $i=0, \ldots, k$. 
In this case

$$
\begin{gathered}
p_{X}(j)=c\left(\begin{array}{l}
k+j \\
k+1
\end{array}\right)-c\left(\begin{array}{c}
k+1-t+j \\
k+1
\end{array}\right)+\left(\begin{array}{c}
k+j \\
k
\end{array}\right)+(c-p)\left(\begin{array}{c}
k+1-t+j \\
k
\end{array}\right), \\
h_{X}(j)= \begin{cases}h_{S}(j) & \text { if } j<t, \\
p_{X}(j) & \text { if } j \geq t,\end{cases} \\
h_{X}(j)-p_{X}(j)+(-1)^{k} h^{k+1}\left(\mathcal{J}_{X}(j)\right)= \begin{cases}p-c & \text { if } j=t-1, \\
0 & \text { otherwise. }\end{cases}
\end{gathered}
$$

Proof. In order to show the first claim we induct on the dimension $k$ of $X$ by applying Lemma 5.3. Condition (a) in that lemma is satisfied by assumption.

Consider a general hyperplane $L$ of $\mathbb{P}^{n}$. Then $X \cap L$ is a divisor on the rational normal scroll $S^{\prime}=S \cap L$ and the divisors $H$ and $F$ restrict to corresponding divisors $H^{\prime}$ and $F^{\prime}$ respectively on $S^{\prime}$. Since condition (b) in Lemma 5.3 is trivially satisfied if $X$ is a curve, Lemma 5.3 implies by induction that $X$ is arithmetically Buchsbaum if and only if

$$
h_{X}(j)-p_{X}(j)=(-1)^{k+1} h^{k+1}\left(\mathcal{J}_{X}(j)\right)
$$

if $j=t$ and if $j=t-k-1$.

In order to check this we compute first using duality and Lemma 5.1

$$
\begin{aligned}
& h^{k+1}\left(S, \mathcal{O}_{S}(-X+j H)\right)=h^{0}\left(S, \mathcal{O}_{S}\left(K_{S}+X-j H\right)\right) \\
& =h^{0}\left(S, \mathcal{O}_{S}((t-k-2-j) H+(2 c-p-1) F)\right) \\
& = \begin{cases}0 & \text { if } j \geq t-k-1, \\
c\left(\begin{array}{c}
t-2-j \\
k+1
\end{array}\right)+(2 c-p)\left(\begin{array}{c}
t-2-j \\
k
\end{array}\right) & \text { if } j \leq t-k-2 .\end{cases}
\end{aligned}
$$

Hence, Lemma 5.5, (b) provides

$$
\begin{aligned}
p_{X}(j) & =p_{S}(j)+(-1)^{k}\left[c\left(\begin{array}{c}
t-1-j \\
k+1
\end{array}\right)+(c-p)\left(\begin{array}{c}
t-2-j \\
k
\end{array}\right)\right] \\
& =p_{S}(j)-c\left(\begin{array}{c}
k+1-t+j \\
k+1
\end{array}\right)+(c-p)\left(\begin{array}{c}
k+1-t+j \\
k
\end{array}\right) .
\end{aligned}
$$

Thus the second claim follows by Lemma 5.4. Moreover, we obtain using Lemma 5.5 and Lemma 5.4

$$
\begin{aligned}
h^{k+1}\left(\mathcal{J}_{X}(j)\right) & =h^{k+1}\left(S, \mathcal{O}_{S}(-X+j H)\right)+ \begin{cases}0 & \text { if } j \geq-k+1, \\
(-1)^{k} p_{S}(j) & \text { if } j \leq-k\end{cases} \\
& = \begin{cases}0 & \text { if } j \geq t-k-1, \\
c\left(\begin{array}{c}
t-1-j \\
k+1
\end{array}\right)+(c-p)\left(\begin{array}{c}
t-2-j \\
k
\end{array}\right) & \text { if }-k+1 \leq j \leq t-k-2, \\
(-1)^{k} p_{X}(j) & \text { if } j \leq-k .\end{cases}
\end{aligned}
$$

Thus we have

$$
\begin{aligned}
h_{X}(t-k-1) & =h_{S}(t-k-1) \quad \text { by Lemma } 5.5, \text { (a) and Lemma } 5.1 \\
& =p_{S}(t-k-1) \quad \text { by Lemma } 5.4 \text { because } t \geq 2
\end{aligned}
$$

and $p_{X}(t-k-1)=p_{S}(t-k-1), h^{k+1}\left(\mathcal{J}_{X}(t-k-1)\right)=0$. Hence, $(*)$ is satisfied if $j=t-k-1$. 
Using the computations above, Lemma 5.5 and Lemma 5.4 we see that for $j=t$ $(*)$ reads as

$$
\begin{aligned}
0 & =h_{X}(t)-p_{X}(t)+(-1)^{k} h^{k+1}\left(\mathcal{J}_{X}(j)\right) \\
& =-h^{0}\left(S, \mathcal{O}_{S}(-X+t H)\right)+c-(c-p)(k+1) \\
& =-h^{0}\left(S, \mathcal{O}_{S}(H+(p-c-1) F)\right)-k c+(k+1) c .
\end{aligned}
$$

According to Lemma 5.1 we obtain

$$
\begin{aligned}
h^{0}\left(S, \mathcal{O}_{S}(H+(p-c-1) F)\right) & =\sum_{j_{0}+\ldots+j_{k}=1} \max \left\{0, p-c+j_{0} a_{0}+\ldots j_{k} a_{k}\right\} \\
& =\sum_{i=0}^{k} \max \left\{0, p-c+a_{i}\right\} \\
& \geq \sum_{i=0}^{k} p-c+a_{i}=(k+1)(p-c)+c
\end{aligned}
$$

where equality holds if and only if $\max \left\{0, p-c+a_{i}\right\}=p-c+a_{i}$ for all $i$. Therefore $(*)$ is satisfied in case $j=t$ if and only if $a_{i} \geq c-p$ for all $i=0, \ldots, k$ proving the first claim.

In this case we get

$$
\begin{aligned}
h^{0}\left(S, \mathcal{O}_{S}(-X+j H)\right) & =h^{0}\left(S, \mathcal{O}_{S}((j-t+1) H+(p-c-1) F)\right) \\
& =\left\{\begin{array}{cl}
0 & \text { if } j<t, \\
c\left(\begin{array}{c}
k+1-t+j \\
k+1
\end{array}\right)+(p-c)\left(\begin{array}{c}
k+1-t+j \\
k
\end{array}\right) & \text { if } j \geq t
\end{array}\right.
\end{aligned}
$$

as in the proof of Lemma 5.1. The difficulty $p-c-1<0$ does not matter because $a_{i} \geq c-p$ ensures that $\max \left\{0, p-c+j_{0} a_{0}+\ldots+j_{k} a_{k}\right\}=p-c+j_{0} a_{0}+\ldots+j_{k} a_{k}$ if $j_{0}+\ldots+j_{k}>0$.

Hence, the computations above and Lemma 5.5, (a) provide our third claim and

$$
\begin{aligned}
& h_{X}(j)-p_{X}(j)+(-1)^{k} h^{k+1}\left(\mathcal{J}_{X}(j)\right) \\
& \quad= \begin{cases}c\left(\begin{array}{l}
k+1-t+j \\
k+1
\end{array}\right)+(p-c)\left(\begin{array}{l}
k+1-t+j \\
k
\end{array}\right) & \text { if } t-k-1 \leq j \leq t-1, \\
0 & \text { otherwise. }\end{cases}
\end{aligned}
$$

The last claim follows.

Lemma 5.9. Let $X \subset \mathbb{P}^{n}$ be a reduced Cohen-Macaulay subscheme being a divisor on a rational normal scroll $S=S\left(a_{0}, \ldots, a_{k}\right)$. Suppose $d>c$ and $X \sim(t+1) H+$ $(1-c-p) F$. Then $X$ is arithmetically Buchsbaum if and only if $a_{i} \geq p$ for all $i=0, \ldots, k$.

In this case

$$
\begin{gathered}
p_{X}(j)=c\left(\begin{array}{l}
k+j \\
k+1
\end{array}\right)-c\left(\begin{array}{c}
k-t+j \\
k+1
\end{array}\right)+\left(\begin{array}{c}
k+j \\
k
\end{array}\right)-p\left(\begin{array}{c}
k-t-1+j \\
k
\end{array}\right), \\
h_{X}(j)= \begin{cases}h_{S}(j) & \text { if } j \leq t \\
p_{X}(j) & \text { if } j \geq t,\end{cases} \\
h_{X}(j)-p_{X}(j)+(-1)^{k} h^{k+1}\left(\mathcal{J}_{X}(j)\right)= \begin{cases}(-1)^{k} p & \text { if } j=t-k, \\
0 & \text { otherwise. }\end{cases}
\end{gathered}
$$


Proof. As in the proof of Lemma 5.8 we see that $X$ is arithmetically Buchsbaum if and only if the condition (*) given in the proof of Lemma 5.8 holds true if $j=t$ and if $j=t-k-1$. In order to check this we compute using Lemma 5.1

$$
\begin{aligned}
h^{0}\left(S, \mathcal{O}_{S}(-X+j H)\right) & =h^{0}\left(S, \mathcal{O}_{S}((j-t-1) H+(c+p-1) F)\right) \\
& = \begin{cases}0 & \text { if } j \leq t, \\
c\left(\begin{array}{c}
j-t-1+k \\
k+1
\end{array}\right)+(c+p)\left(\begin{array}{c}
j-t-1+k \\
k
\end{array}\right) & \text { if } j>t .\end{cases}
\end{aligned}
$$

Then Lemma 5.5, (a) gives

$$
h_{X}(j)= \begin{cases}h_{S}(j) & \text { if } j \leq t \\
h_{S}(j)-c\left(\begin{array}{c}
j-t+k \\
k+1
\end{array}\right)-p\left(\begin{array}{c}
j-t-1+k \\
k
\end{array}\right) & \text { if } j \geq t .\end{cases}
$$

It follows

$$
p_{X}(j)=p_{S}(j)-c\left(\begin{array}{c}
j-t+k \\
k+1
\end{array}\right)-p\left(\begin{array}{c}
j-t-1+k \\
k
\end{array}\right)
$$

proving the second and third claim.

Due to Lemma 5.1 and Lemma 5.5 , (c) we obtain $h^{k+1}\left(\mathcal{J}_{X}(t)\right)=0$. Hence, condition $(*)$ given in the proof of Lemma 5.8 is satisfied if $j=t$. Using the computations above and Lemma 5.5 and Lemma 5.4 this condition $(*)$ reads for $j=t-k-1$ as

$$
\begin{aligned}
0= & h_{X}(t-k-1)-p_{X}(t-k-1)+(-1)^{k} h^{k+1}\left(\mathcal{J}_{X}(t-k-1)\right) \\
= & (-1)^{k}\left[-c+p(k+1)+h^{k+1}\left(S, \mathcal{O}_{S}(-X+(t-k-1) H)\right)\right. \\
& \left.\quad-h^{k+2}\left(\mathcal{J}_{X}(t-k-1)\right)\right] \\
= & (-1)^{k}\left[h^{0}\left(S, \mathcal{O}_{S}\left(K_{S}+X-(t-k-1) H\right)\right)-c+p(k+1)\right] \\
= & (-1)^{k}\left[h^{0}\left(S, \mathcal{O}_{S}(H-(p+1) F)\right)-(c-p(k+1))\right] .
\end{aligned}
$$

Lemma 5.1 yields

$$
\begin{aligned}
h^{0}\left(S, \mathcal{O}_{S}(H-(p+1) F)\right) & =\sum_{j_{0}+\ldots+j_{k}=1} \max \left\{0,-p+j_{0} a_{0}+\ldots+j_{k} a_{k}\right\} \\
& =\sum_{i=0}^{k} \max \left\{0, a_{i}-p\right\} \\
& \geq \sum_{i=0}^{k} a_{i}-p=c-p(k+1)
\end{aligned}
$$

where equality holds if and only if $\max \left\{0, p-c+a_{i}\right\}=a_{i}-p$ for all $i$. Hence, $(*)$ is satisfied in case $j=t-k-1$ if and only if $a_{i} \geq p$ for all $i=0, \ldots, k$ proving the first claim.

Under this assumption we obtain as in the proof of Lemma 5.8

$$
\begin{aligned}
h^{k+1}\left(S, \mathcal{O}_{S}(-X+j H)\right) & =h^{0}\left(S, \mathcal{O}_{S}((t-k-j) H-(p+1) F)\right) \\
& = \begin{cases}0 & \text { if } j \geq t-k, \\
c\left(\begin{array}{c}
t-j \\
k+1
\end{array}\right)-p\left(\begin{array}{c}
t-j \\
k
\end{array}\right) & \text { if } j \leq t-k-1 .\end{cases}
\end{aligned}
$$


Hence, the assumptions above and Lemma 5.5, (c) provide

$$
\begin{aligned}
h_{X}(j) & -p_{X}(j)+(-1)^{k} h^{k+1}\left(\mathcal{J}_{X}(j)\right) \\
= & \begin{cases}c\left(\begin{array}{c}
k-t+j \\
k+1
\end{array}\right)+p\left(\begin{array}{cl}
k-t-1+j \\
k
\end{array}\right) & \text { if } t-k \leq j \leq t-1, \\
0 & \text { otherwise. }\end{cases}
\end{aligned}
$$

The last claim follows.

The main difference in the proofs of the previous two results occurs in the computation of the Hilbert polynomial of $X$. It was crucial to keep the calculations independent of the numbers $a_{i}$, specifying our scroll $S$, as long as possible.

Now we are ready to prove the main result of this paper.

Theorem 5.10. Let $X \subset \mathbb{P}^{n}$ be a reduced Cohen-Macaulay subscheme of dimension $k=n-c>0$ and of degree $d>c$. Write $d=t c+1-p$ where $1 \leq p \leq c$. Suppose $X$ is a divisor on a rational normal scroll $S=S\left(a_{0}, \ldots, a_{k}\right)$. Then

(a) $X$ is arithmetically Cohen-Macaulay if and only if $X \sim t H+(1-p) F$ or $X \sim(t-1) H+F$ provided $c$ divides $d-1$.

(b) $X$ is arithmetically Buchsbaum but not arithmetically Cohen-Macaulay if and only if either

(i) $X \sim(t+1) H+(1-c-p) F$ and $a_{i} \geq p$ for all $i=0, \ldots, k$ or

(ii) $X \sim(t-1) H+(1+c-p) F$ and $a_{i} \geq c-p \geq 1$ for all $i=0, \ldots, k$.

Moreover, there is a smooth arithmetically Buchsbaum variety in any of these divisor classes.

Furthermore, in case (i)

$$
H_{*}^{i}\left(\mathcal{J}_{X}\right) \cong \begin{cases}K^{p}(-t+k) & \text { if } i=k, \\ 0 & \text { if } 1 \leq i<k,\end{cases}
$$

and the homogeneous coordinate ring of $X$ has a minimal free graded resolution of the following form:

$$
0 \rightarrow F_{c+1} \rightarrow \ldots \rightarrow F_{1} \rightarrow R \rightarrow R / I(X) \rightarrow 0
$$

where

$$
F_{i}=R(-1-i)^{\alpha_{i}} \oplus R(-t-i)^{\gamma_{i}} \quad(i=1, \ldots, c+1)
$$

and

$$
\alpha_{i}=i\left(\begin{array}{c}
c \\
i+1
\end{array}\right), \gamma_{i}=c\left(\begin{array}{c}
c-1 \\
i-1
\end{array}\right)+p\left(\begin{array}{c}
c \\
i-1
\end{array}\right) .
$$

In case (ii) we obtain

$$
H_{*}^{i}\left(\mathcal{J}_{X}\right) \cong \begin{cases}K^{c-p}(-t+1) & \text { if } i=1 \\ 0 & \text { if } 2 \leq i \leq k\end{cases}
$$

and the minimal free resolution of $X$ is of the form

$$
0 \rightarrow F_{n} \rightarrow \ldots \rightarrow F_{1} \rightarrow R \rightarrow R / I(X) \rightarrow 0
$$

where

$$
F_{i}=R(-1-i)^{\alpha_{i}} \oplus R(-t+1-i)^{\beta_{i}} \oplus R(-t-i)^{\gamma_{i}} \quad(i=1, \ldots, n)
$$

and

$$
\alpha_{i}=i\left(\begin{array}{c}
c \\
i+1
\end{array}\right)
$$




$$
\begin{gathered}
\beta_{i}= \begin{cases}c-(k+1)(c-p) & \text { if } i=1, \\
0 & \text { if } i>c-(k+1)(c-p),\end{cases} \\
\gamma_{i}= \begin{cases}(c-p)\left[\left(\begin{array}{c}
n+1 \\
i+1
\end{array}\right)-\left(\begin{array}{c}
c \\
i+1
\end{array}\right)\right]-c\left(\begin{array}{c}
c-1 \\
i
\end{array}\right) & \text { if } c-(k+1)(c-p) \leq i<c, \\
(c-p)\left(\begin{array}{c}
n+1 \\
i+1
\end{array}\right) & \text { if } c \leq i \leq n,\end{cases}
\end{gathered}
$$

and

$$
\gamma_{i}-\beta_{i+1}=(c-p)\left[\left(\begin{array}{c}
n+1 \\
i+1
\end{array}\right)-\left(\begin{array}{c}
c \\
i+1
\end{array}\right)\right]-c\left(\begin{array}{c}
c-1 \\
i
\end{array}\right) \quad \text { if } i \geq 1 .
$$

Proof. Let $X \sim \alpha H+\beta F$. Denote the general $(n-k+1)$-section of $X, H, F, S$ by $X^{\prime}, H^{\prime}, F^{\prime}$ and $S^{\prime}$, respectively. Then $X^{\prime}$ is a divisor on the surface scroll $S^{\prime}$ and $X^{\prime} \sim \alpha H^{\prime}+\beta F^{\prime}$. Therefore (a) follows by Lemma 5.6 and Lemma 2.8 and the first claim in (b) is a consequence of Lemma 5.6, Lemma 5.8 and Lemma 5.9.

In order to show the existence claim we follow Harris [11], p. 60. If we let $Y_{0}, \ldots, Y_{k}$ be the homogeneous coordinates on each fibre of $S \cong \mathbb{P}(\mathcal{E}) \rightarrow \mathcal{O}_{\mathbb{P}^{1}}\left(a_{i}\right)$, then a section $\sigma$ of $|\alpha H+\beta F|$ is given by a polynomial

$$
\sigma=\sum_{j_{0}+\ldots+j_{k}=\alpha} \sigma_{j_{0}, \ldots, j_{k}}(z) Y_{0}^{j_{0}} \ldots Y_{k}^{j_{k}}
$$

where $\sigma_{j_{0}, \ldots, j_{k}} \in H^{0}\left(\mathbb{P}^{1}, \mathcal{O}_{\mathbb{P}^{1}}\left(j_{0} a_{0}+\ldots+j_{k} a_{k}+\beta\right)\right)$. In our situation we may write $\alpha=t-s$ and $\beta=1-p+s c$ where $s \in\{1,-1\}$. The assumptions ensure that $j_{0} a_{0}+\ldots+j_{k} a_{k}+\beta>0$ if $j_{0}+\ldots+j_{k}=\alpha$. Hence, for any multi-index $J=j_{0}, \ldots, j_{k}$ the value of the corresponding coefficient function $\sigma_{J}$ may be prescribed at any given value $z$. Therefore the linear system $|\alpha H+\beta F|$ cuts out the subsystem $\left|\mathcal{O}_{\mathbb{P}^{k}}(\alpha)\right|$ on each $k$-plane $\mathbb{P}(\mathcal{E})_{z}$ of $S \cong \mathbb{P}(\mathcal{E})$. Thus $|\alpha H+\beta F|$ has no base points and the generic element of $|\alpha H+\beta F|$ will be smooth and irreducible.

Next, we want to compute the cohomology. In the first case we get by combining Lemma 5.2 and Lemma 5.9 that

$$
\sum_{i=1}^{k}(-1)^{i} h^{i}\left(\mathcal{J}_{X}(j)\right)= \begin{cases}(-1)^{k} p & \text { if } j=t-k \\ 0 & \text { otherwise }\end{cases}
$$

Therefore, the claim is a consequence of Proposition 3.6. In the second case the assertion on the cohomology follows similarly using Lemma 5.8.

In order to get information on the graded Betti numbers of $X$ we use the method developed in the proof of Proposition 2.2. The claims on the length of the resolution follow by the Auslander-Buchsbaum formula and the cohomological characterization of depth. Now put $M=I(X) / I(S)$ and consider the exact sequence

$$
\begin{aligned}
\operatorname{Tor}_{i+1}^{R}(K, M) \rightarrow \operatorname{Tor}_{i}^{R}(K, I(S)) \rightarrow \operatorname{Tor}_{i}^{R}(K, I(X)) \rightarrow \operatorname{Tor}_{i}^{R}(K, M) & \\
& \rightarrow \operatorname{Tor}_{i-1}^{R}(K, I(S)) .
\end{aligned}
$$

According to Lemma 5.8 and Lemma 5.9 we have $a(M) \geq t$; thus $a\left(\operatorname{Tor}_{i}^{R}(K, M)\right) \geq$ $t+i$. Since $\operatorname{Tor}_{i}^{R}(K, I(S)) \cong K(-i-2)^{\alpha_{i-1}}$ (cf., for example, [19], Lemma 2.2) the exact sequence above implies

$$
\operatorname{Tor}_{i}^{R}(K, R / I(X)) \cong K(-i-1)^{\alpha_{i}} \oplus \operatorname{Tor}_{i-1}^{R}(K, M) \quad(i \geq 0) .
$$


Let $X \sim(t+1) H+(1-c-p) F$. Then we know reg $X=t+1$ by Lemma 4.2 and $a(M) \geq t+1$ by Lemma 5.9. Hence, $M$ has a minimal resolution of the form

$$
0 \rightarrow R(-t-n)^{\gamma_{c+1}} \rightarrow \ldots \rightarrow R(-t-1)^{\gamma_{1}} \rightarrow M \rightarrow 0 .
$$

Thus the Hilbert series of $M$ is

$$
H(M, Z)=\frac{\sum_{j=1}^{c+1}(-1)^{j+1} \gamma_{j} Z^{t+j}}{(1-Z)^{n+1}} .
$$

On the other hand we have

$$
H(M, Z)=\frac{\sum_{j \geq 0} \Delta^{k+2} h_{M}(j) Z^{j}}{(1-Z)^{k+2}} .
$$

Due to Lemma 5.4 and Lemma 5.9 we deduce for the Hilbert function of $M$

$$
h_{M}(j)= \begin{cases}0 & \text { if } j \leq t, \\
c\left(\begin{array}{c}
k-t+j \\
k+1
\end{array}\right)+p\left(\begin{array}{c}
k-1-t+j \\
k
\end{array}\right) & \text { if } j>t .\end{cases}
$$

A routine computation gives

$$
\Delta^{k+2} h_{M}(j)= \begin{cases}c+p & \text { if } j=t+1 \\ -p & \text { if } j=t+2 \\ 0 & \text { otherwise }\end{cases}
$$

Comparing the two expressions for the Hilbert series of $M$ we obtain

$$
\sum_{j=1}^{c+1}(-1)^{j+1} \gamma_{j} Z^{t+j}=\left[(c+p) Z^{t+1}-p Z^{t+2}\right](1-Z)^{c-1} .
$$

Now the claim on the Betti numbers follows easily.

Finally, let $X \sim(t-1) H+(1+c-p) F$. We proceed similarly as in the previous case. Unfortunately, the results are less precise.

Due to Lemma 5.4 and Lemma 5.8 we get for the Hilbert function of $M$

$$
h_{M}(j)= \begin{cases}0 & \text { if } j<t, \\
c\left(\begin{array}{c}
k+1-t+j \\
k+1
\end{array}\right)-(c-p)\left(\begin{array}{c}
k+1-t+j \\
k
\end{array}\right) & \text { if } j \geq t .\end{cases}
$$

A lengthy computation provides

$$
\Delta^{k+2} h_{M}(j)= \begin{cases}p-k(c-p) & \text { if } j=t \\
(-1)^{j-t+1}(c-p)\left(\begin{array}{c}
k+2 \\
j-t+1
\end{array}\right) & \text { if } t+1 \leq j \leq t+k+1 \\
0 & \text { otherwise }\end{cases}
$$

Moreover, we already know that $M$ has a minimal free resolution of the form

$$
0 \rightarrow R(-t-n)^{\gamma_{n}} \oplus R(-t-n+1)^{\beta_{n}} \rightarrow \ldots \rightarrow R(-t-1)^{\gamma_{1}} \oplus R(-t)^{\beta_{1}} \rightarrow M \rightarrow 0
$$

where $\beta_{1}=h_{M}(t)=c-(k+1)(c-p) \leq c-2$ because $k \geq 1$ and $p<c$. Since $M$ is torsion-free as $R / I(S)$-module [7], Theorem 1.1 gives $\beta_{i}=0$ if $i>c-(k+1)(c-p)$ and in particular $\beta_{i}=0$ if $i \geq c-1$. Hence, putting $\gamma_{0}=0$ we obtain for the Hilbert series of $M$

$$
H(M, Z)=\frac{\sum_{j=0}^{n}(-1)^{j+1}\left(\gamma_{j}-\beta_{j+1}\right) Z^{t+j}}{(1-Z)^{n+1}} .
$$


Comparison of the two expressions for the Hilbert series of $M$ provides

$$
\begin{aligned}
\sum_{j=0}^{n}(-1)^{j+1}\left(\gamma_{j}-\beta_{j+1}\right) Z^{t+j} & \\
= & {\left[(p-k(c-p)) Z^{t}+\sum_{j=1}^{k+1}(-1)^{j+1}(c-p)\left(\begin{array}{c}
k+2 \\
j+1
\end{array}\right) Z^{t+j}\right](1-Z)^{c-1} } \\
= & (p-k(c-p)) Z^{t}(1-Z)^{c-1} \\
& +(c-p) \sum_{j=1}^{n}\left(\sum_{i=1}^{j}(-1)^{j+1}\left(\begin{array}{c}
k+2 \\
i+1
\end{array}\right)\left(\begin{array}{c}
c-1 \\
j-i
\end{array}\right)\right) Z^{t+j} .
\end{aligned}
$$

It follows for $j \geq 1$

$$
\begin{aligned}
\gamma_{j}-\beta_{j+1}= & -(p-k(c-p))\left(\begin{array}{c}
c-1 \\
j
\end{array}\right) \\
& +(c-p)\left[\left(\begin{array}{c}
k+c+1 \\
j+1
\end{array}\right)-\left(\begin{array}{c}
c-1 \\
j+1
\end{array}\right)-(k+2)\left(\begin{array}{c}
c-1 \\
j
\end{array}\right)\right] \\
= & -(c-(k+1)(c-p))\left(\begin{array}{c}
c-1 \\
j
\end{array}\right) \\
& +(c-p)\left[\left(\begin{array}{c}
n+1 \\
j+1
\end{array}\right)-\left(\begin{array}{c}
c \\
j+1
\end{array}\right)-(k+1)\left(\begin{array}{c}
c-1 \\
j
\end{array}\right)\right] \\
= & (c-p)\left[\left(\begin{array}{c}
n+1 \\
j+1
\end{array}\right)-\left(\begin{array}{c}
c \\
j+1
\end{array}\right)\right]-c\left(\begin{array}{c}
c-1 \\
j
\end{array}\right) .
\end{aligned}
$$

Since we already know $\beta_{1}=c-(k+1)(c-p)$ and $\beta_{i}=0$ if $i>\beta_{1}$, everything is proved.

Remark 5.11. (i) This theorem shows in particular that the divisor class of a reduced arithmetically Buchsbaum scheme $X$ being a divisor on a rational normal scroll is not uniquely determined by its degree and codimension if and only if $X$ is a curve, $c$ is even and $\frac{c}{2}$ divides $d-1$. Whereas the uniqueness result might be surprising, the non-uniqueness result has a simple explanation. Indeed, observe that in this case $p=\frac{c}{2}$ and that the scroll $S$ containing $X$ must be $S\left(\frac{c}{2}, \frac{c}{2}\right)$ which is projectively equivalent to the image of the Segre embedding of $\mathbb{P}^{\frac{c}{2}} \times \mathbb{P}^{\frac{c}{2}}$ in $\mathbb{P}^{c+1}$. The automorphism of $S\left(\frac{c}{2}, \frac{c}{2}\right)$ induced by commuting the factors interchanges the divisor classes $(t+1) H+\left(1-\frac{3 c}{2}\right) F$ and $(t-1) H+\left(1+\frac{c}{2}\right) F$.

(ii) Unfortunately, in case (ii) we cannot always compute all graded Betti numbers. The first open case occurs if $c=4, p=2, k=1$. If $c \leq 3$, then all graded Betti numbers of the possible divisors are covered by Theorem 5.10.

As a consequence of our considerations we obtain that not for any triple $(d, c, k)$ a corresponding Buchsbaum variety, which is not arithmetically Cohen-Macaulay but a divisor on a scroll, does really exist. The precise result is as follows.

Theorem 5.12. Suppose we are given integers $d, c, k$ where $d>c, c \geq 2, k \geq 1$. Write $d=t c+1-p$ where $1 \leq p \leq c$. Then there is an arithmetically Buchsbaum variety $X \subset \mathbb{P}^{c+k}$ of degree $d$, codimension $c$ and dimension $k$ being a divisor on a variety of minimal degree but not arithmetically Cohen-Macaulay if and only if there is a smooth such one if and only if $1 \leq p \leq \frac{c}{k+1}$ or $\frac{k}{k+1} c \leq p<c$. 
Moreover, if $1 \leq p \leq \frac{c}{k+1}$, then such a variety has a minimal free graded resolution of the form described in case (i) of Theorem 5.10.

Proof. Suppose $X \subset \mathbb{P}^{c+k}$ is a divisor on a variety $V \subset \mathbb{P}^{c+k}$ of minimal degree. According to the classification of varieties of minimal degree (cf. [6]) and Proposition $2.9 \mathrm{~V}$ must be a rational normal scroll $S=S\left(a_{0}, \ldots, a_{k}\right)$. Since $a_{0}+\ldots+a_{k}=c$, we get by Theorem 5.10 in case (i) $c \geq(k+1) p$ and in case (ii) $c \geq(k+1)(c-p)$. It follows that the numerical conditions in the statement are necessary. They are also sufficient as is easily seen by Theorem 5.10.

Furthermore, if $1 \leq p \leq \frac{c}{k+1}$, then the considerations above show that

$$
X \sim(t+1) H+(1-c-p) F
$$

unless $k=1$ and $p=\frac{c}{2}$. But then the only other possibility is $X \sim(t-1) H+$ $\left(1+\frac{c}{2}\right) F$. In this case $X$ has the same graded Betti numbers as the varieties in $(t+1) H+\left(1-\frac{3 c}{2}\right) F$ (cf. also Remark 5.11).

With the notation of Theorem 5.12, it turns out that in case $1 \leq p \leq \frac{c}{k+1}$ we have $m(X)=\left\lceil\frac{d}{c}\right\rceil+1$. Hence, it is impossible to strengthen condition (b) in Theorem 4.5 as it was asked in Remark 4.6.

\section{REFERENCES}

[1] E. Ballico, On singular curves in positive characteristic, Math. Nachr. 141 (1989), 267-273. MR 90h: 14042

[2] M. Brodmann, U. Nagel, Bounding cohomological Hilbert functions by hyperplane sections, J. Algebra 174 (1995), 323-348. MR 96d:14017

[3] D. Eisenbud, Commutative algebra with a view toward algebraic geometry, Graduate Texts in Math. 150, Springer-Verlag, 1995. MR 97a:13001

[4] D. Eisenbud, S. Gôto, Linear free resolutions and minimal multiplicity, J. Algebra 88 (1984), 89-133. MR 85f: 13023

[5] D. Eisenbud, J. Harris, Finite projective schemes in linearly general position, J. Algebraic Geom. 1 (1992), 15-30. MR 92i:14035

[6] D. Eisenbud, J. Harris, On varieties of minimal degree (A centennial account), Proceedings of Symposia in Pure Mathematics, Vol. 46 (1987), 3-13. MR 89f: 14042

[7] D. Eisenbud, J. Koh, Some linear syzygy conjectures, Adv. Math. 90 (1991), 47-76. MR 93e: 13019

[8] E. G. Evans, P. Griffith, The syzygy problem, Ann. Math. 114 (1981), 323-333. MR 83i: 13006

[9] L. Gruson, R. Lazarsfeld, C. Peskine, On a theorem of Castelnuovo, and the equations defining space curves, Invent. Math. 72 (1983), 491-506. MR 85g:14033

[10] J. Harris, The genus of space curves, Math. Ann 249 (1980), 191-204. MR 81i:14022

[11] J. Harris, A bound on the geometric genus of projective varieties, Ann. Scuola Norm. Sup. Pisa Cl. Sci. (4) 8 (1981), 36-68. MR 82h:14010

[12] J. Harris, D. Eisenbud, Curves in projective space, Chapter 3, Les Presses de l'Universite, Montreal, 1982. MR 84g:14024

[13] R. Hartshorne, Algebraic geometry, Graduate Texts in Math. 52, Springer-Verlag, 1977. MR 57:3116

[14] C. Huneke, B. Ulrich, General hyperplane sections of algebraic varieties, J. Algebraic Geom. 2 (1993), 487-505. MR 94b:14046

[15] S. Lvovski, On graded Betti numbers for finite subsets of curves, Preprint, Bonn 1997.

[16] D. Mumford, Lectures on curves on an algebraic surface, Ann. of Math. Studies 59, Princeton Univ. Press, 1966. MR 35:187

[17] U. Nagel, On the defining equations and syzygies of arithmetically Cohen-Macaulay varieties in arbitrary characteristic, J. Algebra 175 (1995), 359-372. MR 96f:13023

[18] U. Nagel, On arithmetically Buchsbaum subschemes and liaison, Habilitationsschrift, Paderborn 1996. 
[19] U. Nagel, Y. Pitteloud, On graded Betti numbers and geometrical properties of projective varieties, Manuscripta Math. 84 (1994), 291-314. MR 96d:13018

[20] U. Nagel, P. Schenzel, Degree bounds for generators of cohomology modules and Castelnuovo-Mumford regularity, Nagoya Math. J. (to appear).

[21] U. Nagel, W. Vogel, Bounds for Castelnuovo's regularity and the genus of projective varieties, In: Topics in Algebra. Banach Center Publications, Vol. 26, Part 2, 163-183, PWNPolish Scientific Publishers, Warsaw 1990. MR 93k:13024

[22] J.-P. Serre, Faiscaux algébriques cohérents, Ann. Math. 61 (1955), 197-278. MR 16:953c

[23] J. Stückrad, W. Vogel, Castelnuovo bounds for certain subvarieties in $\mathbb{P}^{n}$, Math. Ann. 276 (1987), 341-352. MR 88e:13013

[24] J. Stückrad, W. Vogel, Buchsbaum rings and applications, Springer-Verlag, 1986. MR 88h:13011a

[25] R. Treger, On equations defining arithmetically Cohen-Macaulay schemes, II, Duke Math. J. 48 (1981), 35-47. MR 84j:14050b

[26] N. V. Trung, G. Valla, Degree bounds for the defining equations of arithmetically CohenMacaulay varieties, Math. Ann. 281 (1988), 209-218. MR 89k:14083

Fachbereich Mathematik und Informatik, Universität-Gesamthochschule Paderborn, D-33095 Paderborn, Germany

E-mail address: uwen@uni-paderborn.de 